\title{
Bioestratigrafía y provincialismo de conodontes del tramo medio-superior de la Formación San Juan en el cerro Viejo de Huaco, Precordillera, Argentina
}

\author{
*Matías J. Mango ${ }^{1}$, Guillermo L. Albanesi ${ }^{1}$ \\ ${ }^{\prime}$ Centro de Investigaciones en Ciencias de la Tierra (CICTERRA), Consejo Nacional de Investigaciones Cientificas y Técnicas \\ (CONICET), Universidad Nacional de Córdoba (UNC), Av. Vélez Sarsfield 1699, X5016GCA, Córdoba, Argentina. \\ matiasjmango@gmail.com; guillermo.albanesi@unc.edu.ar \\ * Autor de correspondencia: matiasjmango@gmail.com
}

\begin{abstract}
RESUMEN. El presente estudio trata sobre la bioestratigrafía de conodontes del tramo medio-superior de la Formación San Juan (Ordovícico Inferior-Medio), en la sección expuesta en la quebrada Los Gatos, ubicada en la vertiente occidental del cerro Viejo de Huaco, Precordillera Central de San Juan. Los conodontes recuperados, correspondientes a 55 especies, permiten reconocer las biozonas en la sección estudiada. El análisis bioestratigráfico posibilita agrupar asociaciones de conodontes asignables a las zonas de Oepikodus evae, Oepikodus intermedius, Baltoniodus triangularis-Tripodus laevis y Lenodus variabilis que corresponden al intervalo Floiano medio-Darriwiliano inferior. Las zonas de Baltoniodus navis y de Microzarkodina parva no se reconocen en esta sección en virtud de la información insuficiente que brinda este tramo estratigráfico. En general, los niveles correspondientes a la Zona de Baltoniodus triangularis-Tripodus laevis contienen mayor cantidad de conodontes, a diferencia de los registros de otras localidades de la Precordillera. El índice de alteración del color de conodontes representa un CAI 2-2,5, refiriendo a paleotemperaturas de soterramiento de los estratos portadores de entre $60^{\circ}$ y $155^{\circ} \mathrm{C}$. La presencia de numerosos conodontes fragmentados, con la lámina superficial recristalizada y con sobrecrecimientos de cristales, indicaría el efecto de procesos fosildiagenéticos distintivos en las calizas portadoras. El análisis de la diversidad y abundancia de géneros y especies de conodontes por intervalos cronoestratigráficos presenta un gran porcentaje de taxones de distribución cosmopolita, un $18 \%$ y $38 \%$ en común a nivel de especie únicamente con la Provincia Norteamericana Midcontinent (PNAM), y un pequeño porcentaje con la Provincia Nord-atlántica (PNA) y endémica de la Precordillera, lo que permite aproximar una mayor afinidad paleobiogeográfica de las asociaciones de conodontes recuperadas con las de la PNAM que con las de la PNA para todo el intervalo estudiado. Por otra parte, se verifica que la Precordillera es una provincia con características propias como la identifican diversos autores.
\end{abstract}

Palabras clave: Bioestratigrafia, Conodontes, Formación San Juan, Ordovícico, Precordillera.

\begin{abstract}
Biostratigraphy and provincialism of conodonts from the middle-upper San Juan Formation in cerro Viejo of Huaco, Precordillera, Argentina. The present study deals with the conodont biostratigraphy from the middle and upper parts of the San Juan Formation (Lower-Middle Ordovician) exposed at the Los Gatos creek section, west of the cerro Viejo de Huaco, Central Precordillera of San Juan Province. The numerous conodonts recovered, corresponding to 55 species, allow to recognize a series of biozones in the studied section. The biostratigraphic analysis carried out herein allows determining associations of conodonts assignable to the Oepikodus evae, Oepikodus intermedius, Baltoniodus triangularis-Tripodus laevis and Lenodus variabilis zones that correspond to the middle Floian to lower Darriwilian. The Baltoniodus navis and Microzarkodina parva zones were not identified due to insufficient information provided by this stratigraphic section. In general, the levels corresponding to the Baltoniodus triangularis-Tripodus laevis Zone contain more conodonts, than the records of other localities from Precordillera. These specimens are well preserved with a color alteration index (CAI) of 2-2.5, indicating overburden paleotemperatures ranging from $60^{\circ}$ and $155^{\circ} \mathrm{C}$. The large presence of fragmented conodonts, with recrystallized surfaces and crystal overgrowth, could indicate the effect of distinctive diagenetic fossil processes on the bearer limestone. The analysis of the diversity and abundance
\end{abstract}


of genera and species of conodonts by chronostratigraphic intervals presents a great percentage of cosmopolitan taxa, $18 \%$ and $38 \%$ in common, at species level, only with the North-American Midcontinent Province (NAMP), and with a small percentage with the North-Atlantic Province (NAP) and the Precordillera, which allows to approximate a greater paleobiogeographic affinity of the conodonts recovered with those of the NAMP than those of the NAP for the entire interval studied. On the other hand, the Precordillera is verified as a province with its own characteristics as identified by several authors.

Keywords: Biostratigraphy, Conodonts, San Juan Formation, Ordovician, Precordillera.

\section{Introducción}

\subsection{Marco geológico}

La Precordillera se localiza en el sector centrooccidental del territorio argentino, limitando al oeste con la Cordillera Frontal y al este con las Sierras Pampeanas. Esta provincia geológica se sitúa entre $\operatorname{los} 28^{\circ} 30^{\prime}$ y $33^{\circ}$ de latitud sur y $\operatorname{los} 68^{\circ} 15^{\prime}$ y $69^{\circ} 45^{\prime}$ de longitud oeste, abarca parte de las provincias de La Rioja, San Juan y Mendoza, y se distingue por afloramientos de rocas paleozoicas muy extensos, en particular los carbonáticos de origen marino y en menor medida los de ambientes continentales del Paleozoico superior, mesozoicos y cenozoicos. La Precordillera puede subdividirse en tres unidades morfoestructurales sobre la base de sus características estratigráficas y tectónicas, las que se conocen como Precordillera Oriental (Ortiz y Zambrano, 1981), Central (Baldis y Chebli, 1969) y Occidental (Baldis et al., 1982). Astini (1992) reconoce una tectofacies oriental mayormente representada por sucesiones de plataforma carbonática cambro-ordovícicas, que incluye a la Precordillera Oriental y Central, y una tectofacies occidental de paleoambientes sedimentarios silicoclásticos profundos, que incluye a la Precordillera Occidental.

Sobre la base del análisis de numerosas evidencias tecto-sedimentarias y de su contenido paleontológico, se ha postulado una posible aloctonía del terreno de Precordillera durante el Paleozoico temprano. Benedetto (1993, 2004) y Astini et al. (1995) proponen el desmembramiento del terreno de Precordillera a partir de los Apalaches, relacionando el arco magmático del Sistema de Famatina con la acreción de la Precordillera a Gondwana durante el Darriwiliano-Sandbiano. Thomas y Astini (1996) y Astini et al. (1996) realizan una síntesis de estas hipótesis, las que proponen que el desprendimiento de la Precordillera provendría de los Apalaches del sur y la región austral de Marathon, Texas, durante el Cámbrico temprano-medio (Ramos,
2004). Finney (2007) postula la hipótesis de un origen para-autóctono de la Precordillera, según la cual, ésta, como parte del terreno de Cuyania, habría migrado a través de una falla transformante desde el margen sur del oeste de Gondwana hacia la posición actual acaecida en el Devónico.

\section{2. Área de estudio}

El perfil estudiado comprende las sedimentitas de la Formación San Juan, aflorantes en el cerro Viejo de Huaco, ubicado al oeste de la localidad de Huaco, Precordillera Central. Las calizas de la Formación San Juan, que constituyen el núcleo del anticlinal de Huaco, se analizan en la quebrada Los Gatos (Figs. 1 y 2), ubicada en el flanco oeste del cerro Viejo de Huaco, aproximadamente a $3 \mathrm{~km}$ al sur de la localidad de La Ciénaga. La referida quebrada es un cauce tributario de la Zanja Honda, con drenaje hacia el río Huaco. En esta sección, el tramo medio-superior de la Formación San Juan se encuentra bien expuesto, permitiendo efectuar estudios bioestratigráficos detallados.

En el cerro Viejo de Huaco, se ha documentado la bioestratigrafía de su parte inferior (Lemos, 1981; Di Prinzio y Hünicken, 1990; Mestre, 2008; Mango et al., 2016) y su techo (Ortega, 1987; Ottone et al., 1999; Ortega et al., 2007; Mestre et al., 2013), no así su tramo medio-superior que aquí se analiza, estudiado previamente en las localidades de Pachaco (Serpagli, 1974) y del cerro Potrerillo (Albanesi et al., 1998), en la sección de Niquivil (Lehnert, 1995; Albanesi et al., 2006), en el área comprendida por los ríos Guandacol y Gualcamayo (Albanesi et al., 1999) de la Precordillera Central, y en secciones de la sierra de Villicum (Sarmiento, 1987; Mestre, 2013) de la Precordillera Oriental. En trabajos previos sobre el provincialismo de conodontes (Serpagli, 1974; Bagnoli y Stouge, 1991; Albanesi y Bergström, 2004, 2010) se analizan los registros específicos de diversas localidades de la Precordillera. 


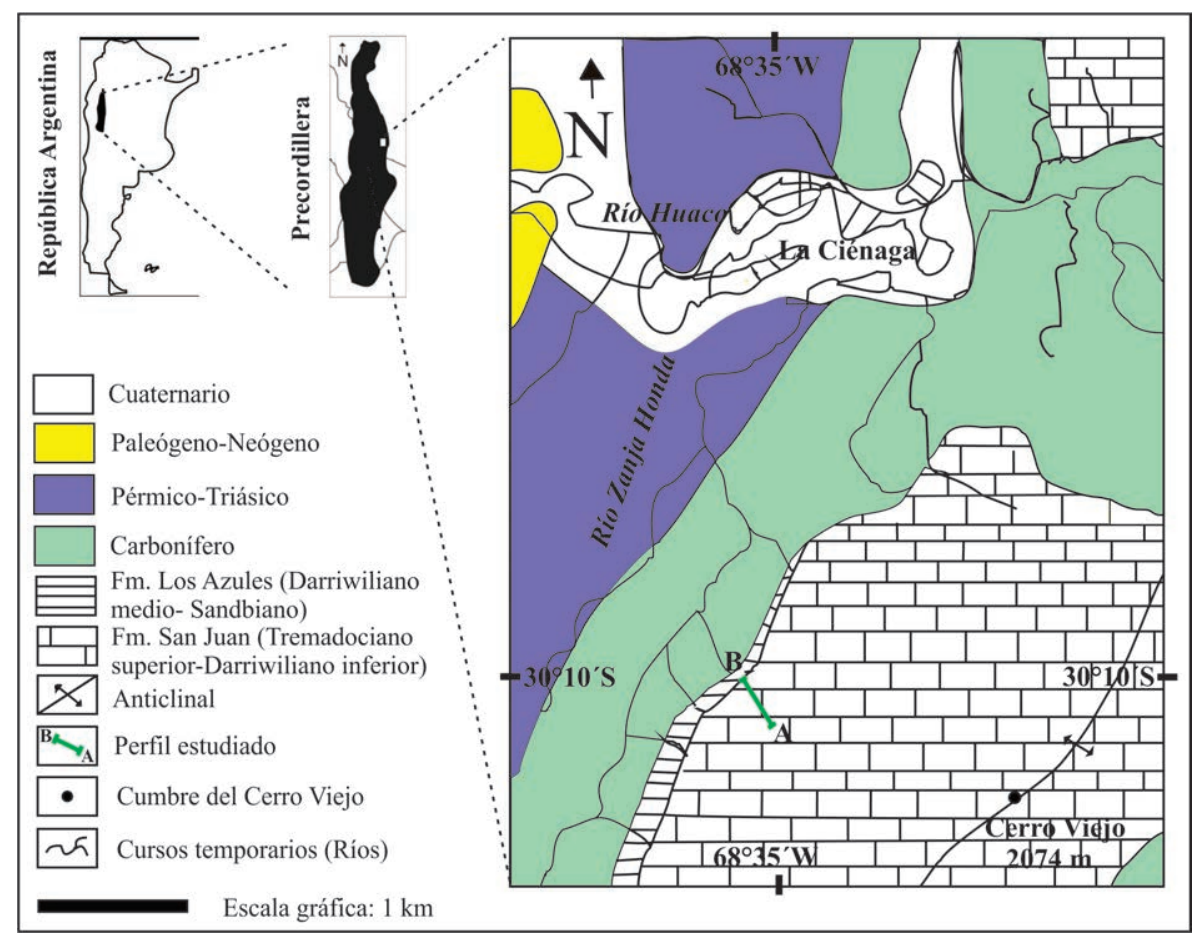

FIG. 1. Mapa geológico del área de estudio y localización de la sección estratigráfica analizada. Referencias del perfil: A: inicio; B: techo de la Fm. San Juan (modificado de Ortega et al., 2007).

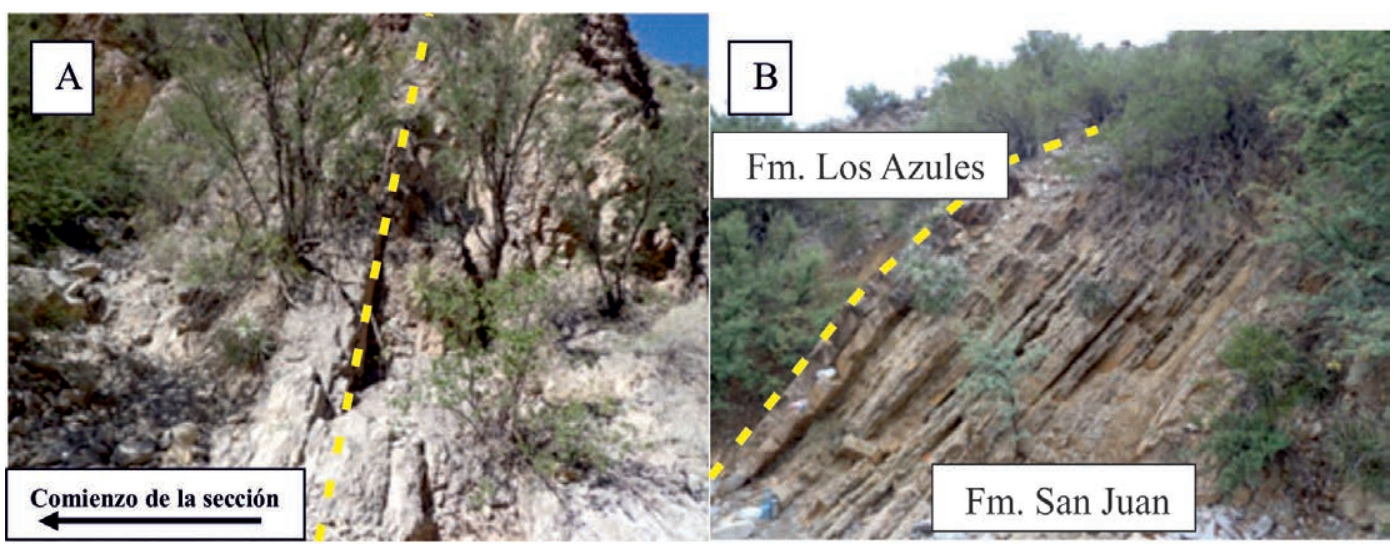

FIG. 2. A. Nivel basal de la sección estratigráfica de la Formación San Juan delimitado por la línea punteada (caminando aproximadamente $424 \mathrm{~m}$ por debajo del techo) (vista al norte); B. Techo de la Formación San Juan en la quebrada Los Gatos, cerro Viejo de Huaco, marcado por la línea punteada, observándose el contacto paraconcordante con el Miembro Inferior de la Formación Los Azules (vista al norte).

El objetivo de este trabajo es estudiar la fauna de conodontes del tramo medio-superior de la Formación San Juan en la sección de la quebrada de Los Gatos, para determinar las características y diferencias del registro taxonómico y paleobiogeográfico a través de la cuenca. En el mismo se trata de determinar el predominio de la afinidad paleobiogeográfíca y si esta se modifica a través del tiempo. Para ello se analiza 
la asociación de conodontes recuperada y se evalúa la distribución de géneros y especies registrados para el intervalo Floiano medio-Darriwiliano temprano, comparándola con la Provincia Norteamericana Midcontinent (PNAM) y con la Provincia Nordatlántica (PNA).

\section{Estratigrafía}

\subsection{La Formación San Juan}

La sucesión carbonática de la Formación San Juan (Kobayashi, 1937), de aproximadamente $330 \mathrm{~m}$ de espesor, está constituida por calizas micríticas esqueletales depositadas a partir de Tremadociano tardío sobre una topografía de rampa, registrándose dos ciclos regresivos-transgresivos (Cañas, 1995; Carrera et al., 2013).

El contacto entre la Formación La Silla y la suprayacente Formación San Juan, marca un cambio mayor en la conación de la plataforma carbonática cambro-ordovícica, con el pasaje de facies de ambiente submareal a facies de plataforma abierta y geometría de rampa carbonática (Pratt et al., 2012).

Las calizas de la Formación San Juan se inician con una secuencia transgresiva en cuya base se desarrolla un horizonte arrecifal constituido por calcimicrobios, esponjas, crinoideos y algas bentónicas (Cañas y Keller, 1993; Cañas y Carrera, 2003). Estos se presentan como montículos de alrededor de $4 \mathrm{~m}$ de espesor y varios cientos de metros de extensión areal. Sucesivamente se acumulan sedimentos que originan depósitos de mar alto (mayormente wackestone esqueletales bioturbados) en un marco de estabilidad ambiental, permitiendo el desarrollo de ricas comunidades submareales dominadas por organismos suspensívoros, especialmente braquiópodos y gastrópodos macluritáceos (Cech y Carrera, 2002).

Un segundo horizonte arrecifal, que se presenta con montículos de $10 \mathrm{~m}$ de ancho y $5 \mathrm{~m}$ de espesor, separados entre 10 y $100 \mathrm{~m}$, formados por microbialitas, receptaculítidos (Calathium) y principalmente por estromatoporoideos (Zondarella), se desarrolla cerca de la base del Ordovícico Medio. En las calizas correspondientes a la secuencia depositacional superior, los braquiópodos son el grupo de invertebrados dominante, solo equiparados en importancia por la diversificación de las demosponjas (Carrera, 2003).
Durante el Darriwiliano, como consecuencia de un aumento relativo del nivel del mar que sumergió a la rampa carbonática de la Formación San Juan por debajo de la zona fótica, se produce la sofocación de la producción de carbonatos y la culminación del ciclo carbonático con la posterior depositación de la facies calcáreo-pelítica transicional hacia secuencias predominantemente pelíticas (Baldis y Beresi, 1981; Baldis et al., 1989) conocidas como formaciones Gualcamayo, Los Azules, Las Aguaditas y equivalentes estratigráficos laterales, en diversas localidades de la Precordillera.

Entre la Formación San Juan y la suprayacente Formación Los Azules, se documenta una superficie costrificada de tipo hardground, interpretándose el contacto como una paraconformidad (Astini, 1994). Éste representaría un hiatus breve, que Ortega et al. (1996) consideran difícil de acotar, por superar el potencial de resolución de la bioestratigrafía clásica.

\subsection{Antecedentes bioestratigráficos de conodontes en el área de estudio}

Lemos (1981) estudia el tramo superior de la Formación San Juan en el Anticlinal de Huaco (sección de Buenaventura Luna), registrando las especies Baltoniodus navis (Lindström), Drepanodus arcuatus Pander, Juanognathus jaannussoni Serpagli, J. variabilis Serpagli, Paroistodus parallelus (Pander), Periodon flabellum (Lindström), Protopanderodus elongatus Serpagli, Reutterodus andinus Serpagli, donde la presencia de Baltoniodus navis (Lindström) permitiría reconocer la biozona homónima. Di Prinzio y Hünicken (1990) documentan la Zona de Oepikodus evae en la parte inferior y media (156 m de espesor), y por encima las zonas de $O$. intermedius y O. communis ( $72 \mathrm{~m}$ de espesor); si bien estas últimas unidades son inferidas por correlación regional. Posteriormente, Mestre (2008) realiza una revisión bioestratigráfica de los conodontes del tope de la Formación San Juan en el sector del Monumento a Buenaventura Luna, reconociendo la Zona de Oepikodus evae en contraposición con los estudios previamente mencionados y Mango et al. (2016) registran la Zona de Prioniodus elegans en los estratos más bajos aflorantes y la Zona de Oepikodus evae en su techo, así como en los dropstones contenidos en los estratos basales de la Formación Guandacol (Carbonífero), que suprayace mediante discordancia erosiva a la Formación San Juan. 
Hünicken y Ortega (1987) analizan los estratos cuspidales de la Formación San Juan en el cerro Viejo de Huaco, y recuperan especímenes que atribuyen a Eoplacognathus suecicus Bergström con la identificación de la biozona homónima. Ottone et al. (1999) estudian la bioestratigrafía de palinomorfos, graptolitos y conodontes del techo de la Formación San Juan y de toda la Formación Los Azules, en diversas quebradas del cerro Viejo de Huaco (Los Gatos, Los Azules, Amarilla y El Silencio) y registran conodontes de la Zona de Lenodus variabilis en el techo de la Formación San Juan, revisando los registros previos de Hünicken y Ortega (1987). Ottone et al. (1999) determinan una fauna de conodontes que incluye, de acuerdo con la actualización taxonómica más reciente, a: Ansella jemtlandica (Löfgren), "Bryantodina" aff. typicalis Stauffer, Cornuodus longibasis (Lindström), Costiconus costatus (Dzik), Drepanodus reclinatus (Lindström), Drepanoistodus basiovalis (Sergeeva), D. bellburnensis Stouge, D. costatus (Abaimova), D. tablepointensis Stouge, Erraticodon alternans (Hadding), Fahraeusodus jachalensis Feltes y Albanesi, Lenodus variabilis (Sergeeva), Oistodus striolatus Serpagli, Paltodus? jemtlandicus Löfgren, Parapaltodus simplicissimus Stouge, Paroistodus horridus (Barnes y Poplawski), $P$. originalis (Sergeeva), Periodon flabellum Lindström, Protopanderodus gradatus Serpagli, P. robustus (Hadding), Pteracontiodus cryptodens (Mound), Rossodus barnesi Albanesi, Yansodus bernabei Albanesi, y Spinodus spinatus (Hadding).

Los estudios recientes (Heredia y Mestre, 2011; Feltes et al., 2013; Serra et al., 2013) posibilitaron la actualización de los esquemas bioestratigráficos de Precordillera, proponiendo la Zona de Yangzeplacognathus crassus para la porción superior de la Zona de Lenodus variabilis. Mestre et al. (2013) analizan los conodontes asociados a espículas de esponjas y foraminíferos del techo de la Formación San Juan en la quebrada Del Aluvión (cerro Viejo de Huaco), registrando la Zona de Yangtzeplacognathus crassus. Ortega et al. (2007) ilustran al conodonte epónimo de la Zona de Yangzeplacognathus crassus como $L$. variabilis para la quebrada de Los Azules (Albanesi y Ortega, 2016). Según estos autores, la presencia de Paroistodus $h$. horridus confirma la biozona en cuestión, en las secciones situadas hacia el sur de la quebrada de Los Gatos, en el flanco occidental del cerro Viejo de Huaco, lo cual revela el carácter diacrónico del techo de la Formación San Juan (Hünicken, 1985).

\section{Materiales y Métodos}

Las tareas de campo comprendieron el reconocimiento de la geología del área de estudio y el levantamiento de un perfil estratigráfico correspondiente al tramo medio-superior de la Formación San Juan en la quebrada de Los Gatos, cerro Viejo de Huaco. Se tomaron 26 muestras de calizas de $1 \mathrm{a} 4 \mathrm{~kg}$, para la recuperación de conodontes a intervalos regulares de $20 \mathrm{~m}$ de espesor aparente, y se documentó la sección estudiada mediante fotografías de campo.

En el laboratorio se procesaron las rocas para la recuperación de los microfósiles, siguiendo el método de Stone (1987) (ácido acético glacial al 10\%). Por cada muestra procesada se obtuvo entre 20 y $150 \mathrm{~g}$ de residuo insoluble, según la composición de la caliza, del que se recuperaron mediante picking 668 conodontes, que fueron montados en portaobjetos.

Los conodontes determinados se ilustraron mediante fotomicrografía óptica convencional y microscopía electrónica de barrido, con instrumentos Leica DMLP y Zeiss, respectivamente. El índice de alteración del color se determinó a través de colecciones de referencia y cartas de colores estandarizadas. Las ilustraciones y gráficos analíticos se confeccionaron mediante programas de computación específicos.

\section{Características de los conodontes recuperados}

Los conodontes presentan una variación de color, producida por la temperatura y el tiempo de soterramiento; este se expresa mediante el índice de alteración del color (Color Alteration Index-CAI) (Epstein et al., 1977), que relaciona los colores con las temperaturas que representan. Los conodontes recuperados de la Formación San Juan corresponden a un valor de CAI 2-2,5, que indicaría una paleotemperatura de soterramiento de entre 60 y $155^{\circ} \mathrm{C}$ (Epstein et al., 1977). La presencia de numerosos conodontes fragmentados, con la lámina superficial recristalizada y con sobrecrecimientos de cristales, refleja la actuación de los procesos fosildiagenéticos en calizas, causantes de las alteraciones superficiales que manifiestan estos microfósiles.

\section{Bioestratigrafía de conodontes}

En este trabajo se sigue el reglamento del Comité Argentino de Estratigrafía (1992) y el esquema 
bioestratigráfico para el Sistema Ordovícico propuesto por Albanesi y Ortega $(2002,2016)$ para la Precordillera Argentina (Fig. 3), reconociéndose las zonas de Oepikodus evae, Oepikodus, Oepikodus intermedius, Baltoniodus triangularis-Tripodus laevis y Lenodus variabilis, para el perfil estratigráfico analizado.

\subsection{Zona de Oepikodus evae}

Albanesi et al. (1998) definen esta biozona en la Formación San Juan, para la sección de Portezuelo Yanso. El límite inferior de la biozona estaría indicado por el registro más bajo de la especie nominal y el límite superior coincidiría con la última aparición de la misma. Esta zona es una biozona de intervalo basada en un taxón y corresponde al Floiano mediosuperior.

En la Zona de Oepikodus evae, los autores reconocen dos subzonas; una inferior, denominada
Subzona de Oepikodus evae-Juanognathus variabilis (Subbiozona de asociación) y una superior, la Subzona de Oepikodus evae-Scolopodus oldstockensis (Subbiozona de asociación).

El límite inferior de la Subzona de Oepikodus evae-Juanognathus variabilis corresponde al límite inferior de la zona y el límite superior estaría dado por la primera aparición de Scolopodus oldstockensis Stouge.

El límite inferior de la Subzona de Oepikodus evae-Scolopodus oldstockensis coincide con el primer registro de Scolopodus oldstockensis Stouge y el límite superior corresponde al límite análogo de la biozona.

Observaciones en el área de estudio y discusión: la presencia de la especie $O$. evae, que da nombre a la biozona, se registra desde la muestra LG 2 hasta la muestra LG 6 (Figs. 4, 5 y 6). El límite inferior de la biozona permanece abierto y se ubica tentativamente en el nivel LG 2 por corresponderse con los primeros

\begin{tabular}{|c|c|c|c|c|c|c|c|}
\hline \multirow{2}{*}{ 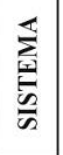 } & \multirow{2}{*}{ 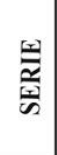 } & \multirow{2}{*}{$\stackrel{\circ}{2}$} & \multirow{2}{*}{ 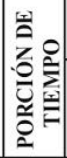 } & \multicolumn{4}{|c|}{ CONODONTES } \\
\hline & & & & NORTEAMÉRICA & $\begin{array}{c}\text { BALTO- } \\
\text { ESCANDINAVIA }\end{array}$ & $\begin{array}{l}\text { PRECORDILLERA } \\
\text { ARGENTINA }\end{array}$ & $\begin{array}{c}\text { NOROESTE } \\
\text { ARGENTINA }\end{array}$ \\
\hline \multirow{16}{*}{$\underset{\cup}{0}$} & \multirow{10}{*}{$\frac{0}{2}$} & \multirow{7}{*}{ 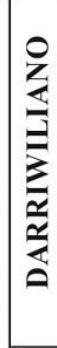 } & \multirow{4}{*}{ 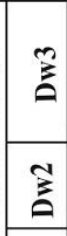 } & sweeti & $\begin{array}{r}\text { anserinus kiel. } \\
\text { lind. }\end{array}$ & anserinus low. & \multirow{6}{*}{ "Erismodus, } \\
\hline & & & & friendsvillensis & \multirow{2}{*}{$\begin{array}{l}\text { serra } \frac{r e c .}{\text { fol. }} \\
\text { suecicus }\end{array}$} & $\frac{\frac{l i n d}{r o b} .}{\frac{r e c}{f o t}}$ & \\
\hline & & & & \multirow[t]{2}{*}{ polonicus } & & suecicus $\frac{a m i}{k r i}$ & \\
\hline & & & & & pseudoplanus & pseudoplanus $\frac{\sigma z}{h a}$ & \\
\hline & & & & holodentata & crassus & crassus & \\
\hline & & & \multirow{2}{*}{ 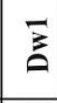 } & \multirow{2}{*}{ sinuosa } & variabilis & variabilis $\frac{\text { ho. }}{\text { gla. }}$ & \\
\hline & & & & & antivariabilis & \multirow{2}{*}{ parva } & \\
\hline & & \multirow{3}{*}{ 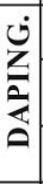 } & ڤ & \multirow{2}{*}{ altifrons } & norrlan./parva & & \\
\hline & & & \multirow{2}{*}{ 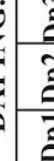 } & & Originalus & navis & “B. navis" \\
\hline & & & & $\begin{array}{l}\text { flabellum/ } \\
\text { laevis }\end{array}$ & triangularis & triang./laevis & B. triang. \\
\hline & \multirow{6}{*}{\multicolumn{2}{|c|}{$=$}} & \multirow{3}{*}{\begin{tabular}{l|}
$\frac{m}{I}$ \\
$\frac{2}{x}$ \\
\end{tabular}} & \multirow{3}{*}{ andinus } & \multirow{3}{*}{ evae } & intermedius & B. cf. triang. \\
\hline & $\underline{\underline{a}}$ & & & & & olds. & T. diprion \\
\hline & $\underline{a}$ & & & & & var: & G. andinus \\
\hline & 도 & & & \multirow{3}{*}{ communis } & \multirow[b]{2}{*}{ elegans } & \multirow{3}{*}{ elegans } & G. vetus \\
\hline & & & \multirow[t]{2}{*}{$\overline{\mathrm{C}}$} & & & & \multirow{2}{*}{ A. triang. } \\
\hline & & & & & elong./delt. & & \\
\hline
\end{tabular}

FIG. 3. Esquema cronobioestratigráfico del Floiano-Darriwiliano (Ordovícico Inferior-Medio), con zonaciones de conodontes de Norteamérica y de Baltoescandinavia, y biozonas de la Precordillera y el noroeste argentino. Se recuadran las biozonas correspondientes al intervalo que se discute en este estudio (modificado de Albanesi y Ortega, 2016). 


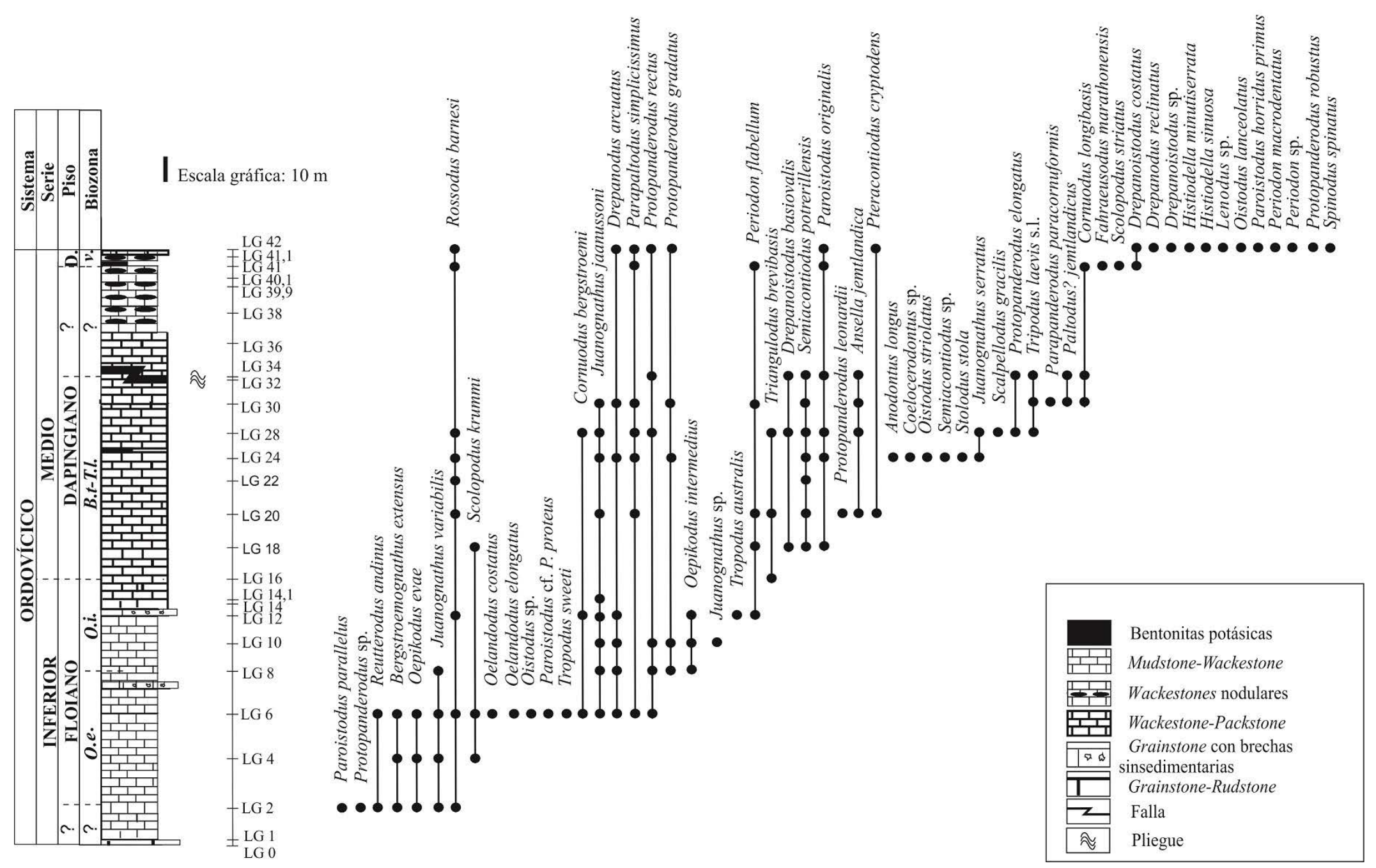

FIG. 4. Columna estratigráfica del tramo medio-superior de la Formación San Juan en la quebrada de Los Gatos, con la distribución vertical de las especies de conodontes recuperados. D.: Darriwiliano; O.e.: Oepikodus evae; O.i.: Oepikodus intermedius; B.t-T.l.: Baltoniodus triangularis-Tripodus laevis; v.: Lenodus variabilis. 

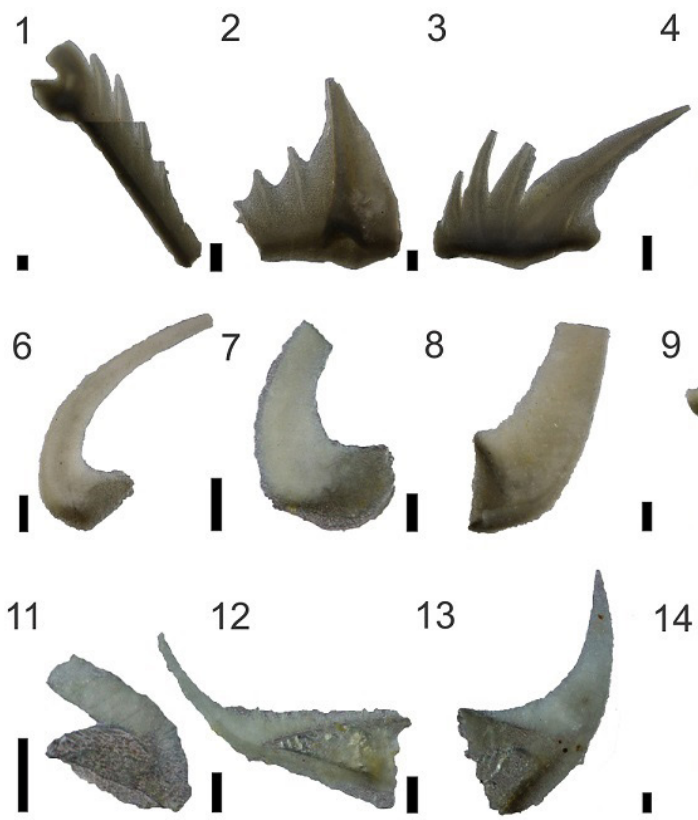

4

8

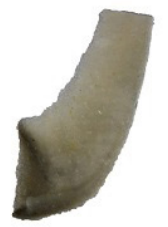

9

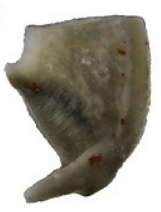

10
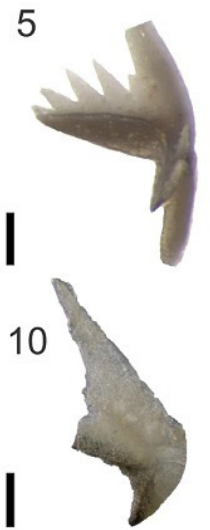

13

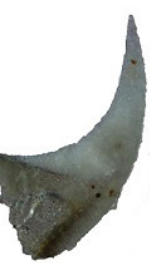

14

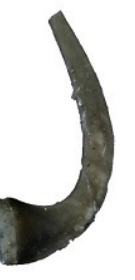

15

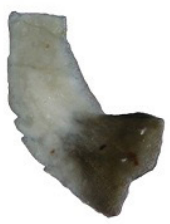

16
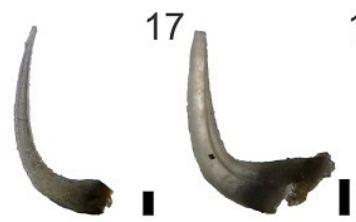

18

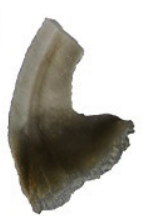

19

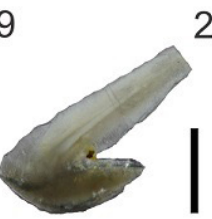

20

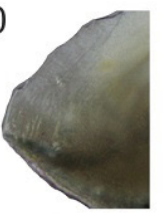

21

22
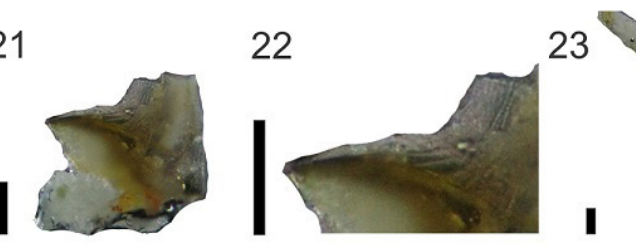

24
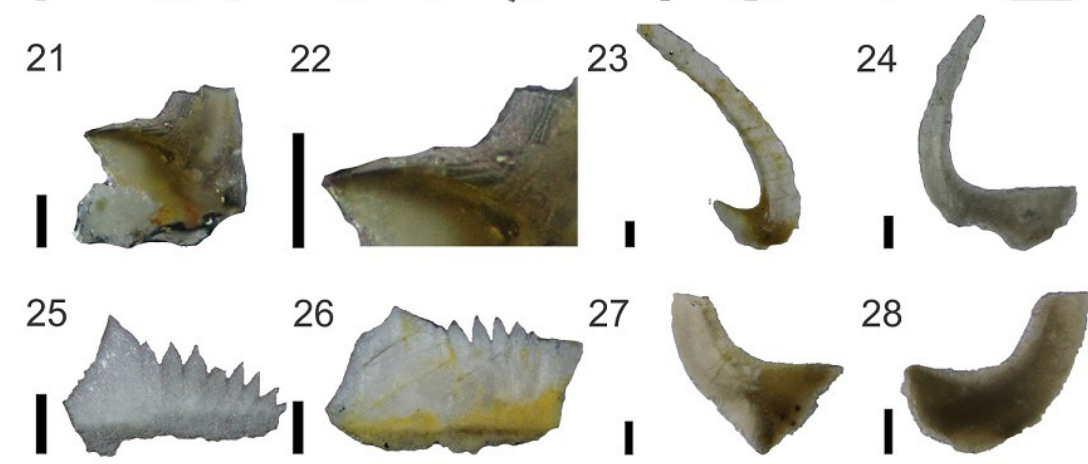

27
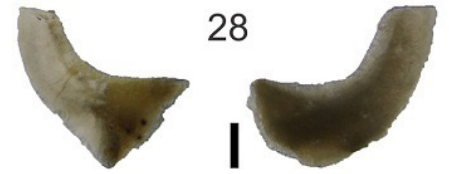

FIG. 5. 1-9. Zona de Oepikodus evae: 1-3. Bergstroemognathus extensus Serpagli; 1. M, LG 6, CORD-MP 56563; 2. Sa, LG 6, CORD-MP 56564; 3. P, LG 6, CORD-MP 56565; 4-5. Oepikodus evae (Lindström); 4. Sb, LG 6, CORD-MP 56566; 5. Pb, LG 6, CORD-MP 56567; 6. Paroistodus parallelus (Pander), S, LG 2, CORD-MP 565683; 7. Paroistodus cf. P. proteus (Lindström), S, LG 6, CORD-MP 56569; 8-9. Reutterodus andinus Serpagli; 8. M, LG 6, CORD-MP 56570; 9. P, LG 2, CORDMP 56571. 10. Zona de Oepikodus intermedius: 10. Tropodus sweeti (Serpagli), Sb, LG 6, CORD-MP 56572. 11-18. Zona de Baltoniodus triangularis-Tripodus laevis: 11-13. Ansella jemtlandica (Löfgren); 11. M, LG 34, CORD-MP 56573; 12. Sa, LG 30, CORD-MP 56574; 13. P, LG 28, CORD-MP 56575; 14. Anodontus longus Stouge y Bagnoli, LG 24, CORD-MP 56576; 15. Juanognathus serratus (Xiang y Zhang), b, LG 28, CORD-MP 56577; 16-18. Semiacontiodus potrerillensis Albanesi; 16. a, LG 24, CORD-MP 56578; 17. c, LG 30, CORD-MP 56579; 18. f, LG 34, CORD-MP 56580. 19-28. Zona de Lenodus variabilis: 19-22. Drepanoistodus costatus Abaimova; 19. M, LG 42 , CORD-MP 56581; 20. estrías del margen antero basal del elemento ilustrado en la imagen 19, LG 42; 2.1. Sa, LG 41, CORD-MP 56582; 22. estrías del margen postero basal del elemento ilustrado en la imagen 21, LG 41; 23-24. Drepanodus reclinatus (Lindström); 23. c, LG 42, CORD-MP 56583; 24. e, LG 42, CORD-MP 56584; 25. Histiodella minutiserrata (Mound), Pb, LG 42 , CORD-MP 56585; 26. Histiodella sinuosa (Graves y Ellison), Pa, LG 42, CORD-MP 56586; 27-28. Protopanderodus robustus (Hadding); 27. a-b, LG 42, CORD-MP 56587; 28. c, LG 42, CORD-MP 56588. Escala gráfica. $100 \mathrm{~mm}$. 


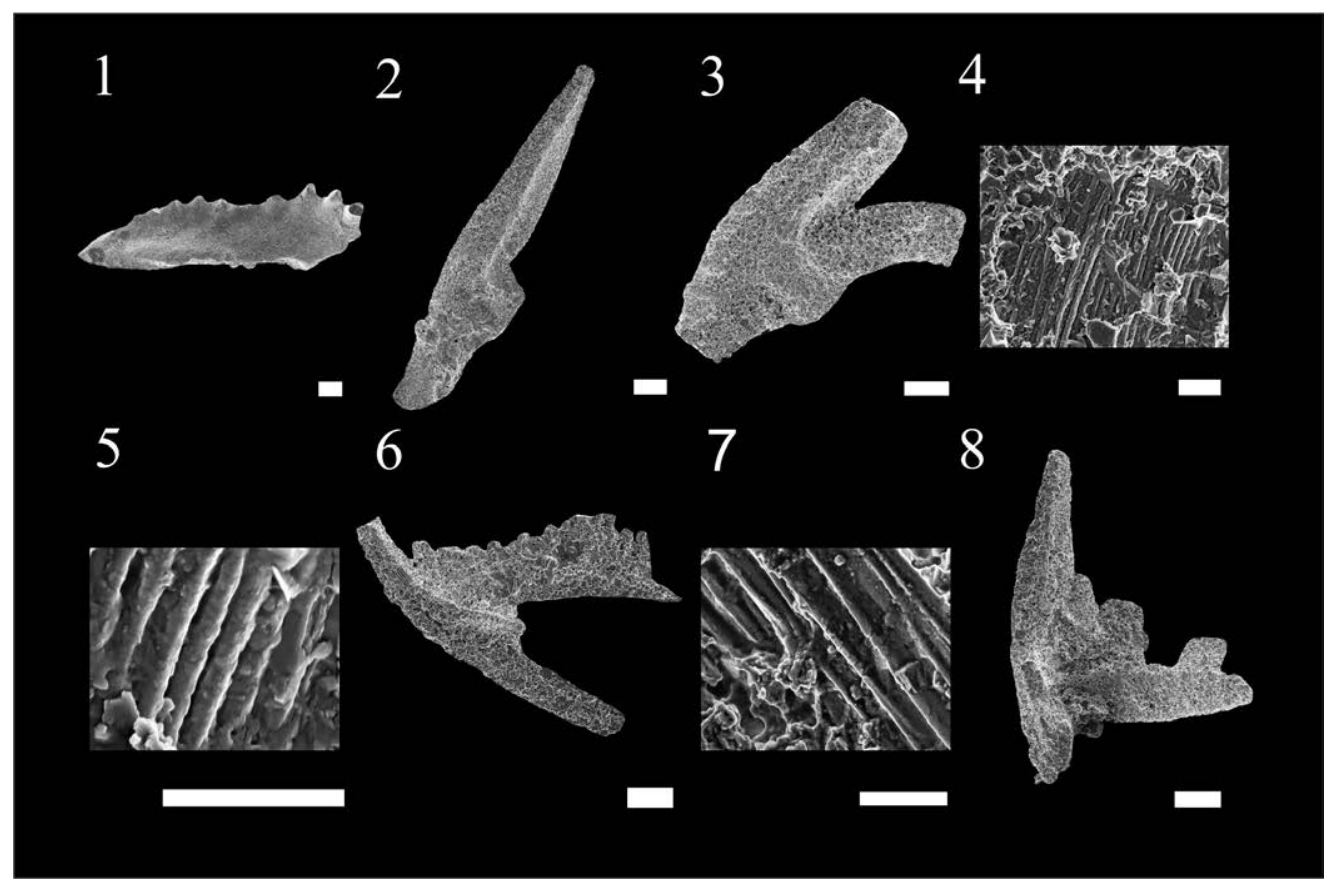

FIG. 6. 1. Lenodus sp., fragmento de elemento P, LG 42, CORD-MP 56589; 2-8. Oepikodus evae (Lindström); 2-3. M, LG 6, CORD-MP $56590 / 1 ; 4$. estrías presentes en la cúspide, observables en el elemento de la figura 3, LG 6; 5. estrías de la cúspide, observables en el elemento de la figura 3, LG 6; 6. Sd, LG 6, CORD-MP 56592; 7. estrías de la cúspide, observables en el elemento de la figura 6, LG 24; 8. Pb, LG 6, CORD-MP 56593. Escala gráfica. $50 \mathrm{~mm}$.

niveles productivos de la referida especie. El límite superior de la biozona reconocida estaría situado en el nivel LG 8, indicado por la aparición en el registro de $O$. intermedius Serpagli, en ausencia de $O$. evae (Lindström).

En la Zona de Oepikodus evae, Subzona de O. evae-Juanognathus variabilis, la especie $J$. variabilis presenta su acmé, y en este trabajo se registra de manera abundante. Considerando el rango que presenta Oepikodus evae, se podría estimar que el intervalo comprendido entre los niveles LG 2 y LG 6 correspondería a la Subzona de O. evae-J. variabilis; sin embargo, debido a la ausencia de un registro fósil más completo que permita una delimitación más precisa de las subzonas, se sugiere esta aproximación bioestratigráfica.

Entre las muestras LG 2 y LG 8 del perfil son comunes las especies Bergstroemognathus extensus Serpagli (Fig. 5), Scolopodus krummi (Lehnert) y Juanognathus variabilis, además, se registraron los únicos elementos de Oelandodus costatus van Wamel, Oelandodus elongatus (Lindström), Paroistodus parallelus (Pander), Paroistodus cf.
P. proteus (Lindström), Reutterodus andinus Serpagli y Tropodus sweeti (Serpagli).

Correlación regional: la fauna B de Serpagli (1974) para la Formación San Juan de la sección de Pachaco, sobre el río San Juan, Precordillera, es equivalente a la Zona de Oepikodus evae identificada en este trabajo.

Lehnert $(1993,1995)$ establece la Zona de O. evae y de O. evae./O. intermedius en la sección de Niquivil de la Precordillera Central de San Juan donde aflora la Formación San Juan, y Lehnert et al. (1998) la identifican en parte del tramo medio de la Formación Ponón Trehué. Estas se correlacionan con la Zona de O. evae establecida por Albanesi et al. (1998) para la Formación San Juan en el cerro Potrerillo.

Mestre (2008) y Mango et al. (2016), como se mencionó previamente, reconocieron esta biozona en la Formación San Juan en el sector del Monumento a Buenaventura Luna situado en la provincia de San Juan.

En la Formación Suri, del Sistema de Famatina, se registra la especie guía que permite asignarla a esta biozona (Lehnert et al., 1997; Albanesi y Astini, 2000). 
En la sección de Niquivil de la Precordillera Central de San Juan y de Peña Sombría de la Precordillera de La Rioja, Albanesi et al. (2006) documentan la Zona de O. evae.

La Zona de Oepikodus evae fue reconocida, además, en la quebrada de Talacasto, Precordillera Central de San Juan, por Soria et al. (2013), donde los autores recuperaron conodontes presentes en mudstones de la Formación San Juan.

Carlorosi y Heredia (2013) analizan muestras obtenidas de la Formación Acoite, Cordillera Oriental, Noroeste de Argentina, y registran la Zona de Trapezognathus diprion; la parte inferior de esta unidad se correlacionaría con el tramo superior de la Zona de O. evae de Albanesi et al. (1998).

Estratos calcáreos interestratificados en la Formación Cieneguillas del área de Purmamarca y Lipán, Cordillera Oriental de Jujuy, posee una baja diversidad de conodontes que se correlacionaría con la Zona de Oepikodus evae (Rao et al., 1994; Albanesi et al., 2014).

Voldman et al. (2017) establecen la Zona de Gothodus andinus, en el tramo superior de la Formación Acoite en la sección de la quebrada Chulpíos, Santa Victoria, Cordillera Oriental de Salta. El tramo superior de la misma podría correlacionarse con la Zona de O. evae (Subzona de Oepikodus evaeJuanognathus variabilis) siguiendo a Albanesi y Ortega (2016).

En el tramo inferior de la Formación San José en la sección Cárcel Punco, Cordillera Oriental de Perú, Gutiérrez-Marco et al. (2008) registran una conodontofauna con elementos que permiten identificar la Zona de $O$. evae (como por ejemplo Trapezognathus diprion), aunque no la especie guía.

Correlación intercontinental: la Zona de Oepikodus evae se puede correlacionar con la parte media-superior de la Zona de Oepikodus communis en los esquemas bioestratigráficos de Norteamérica (Ethington y Repetski, 1984). En el este y norte de Groenlandia, Smith (1991) define la Zona de Oepikodus communis, la cual sería equivalente a la zona homónima de Ethington y Repetski (1984), con una extensión cronoestratigráfica que comprendería la Zona de Paroistodus proteus (límite TremadocianoFloiano) en el esquema bioestratigráfico de la Precordillera argentina.

Pohler y Orchard (1990) analizan conodontes provenientes de la cuenca de Kechika, Canadá, registrando a Tropodus sweeti y a Parapanderodus arcuatus Stouge, por lo que asignan tentativamente estos niveles a la Zona de Oepikodus evae. Conodontes de la Zona de Oepikodus evae han sido identificados en rocas de la Formación Skoki, en el área de Ware, noreste de la Columbia Británica (Pyle y Barnes, 2002).

La Formación Broken Skull en el área de Nahanni, oeste de la Cordillera de Canadá, presenta conodontes típicos de la Zona de Oepikodus evae, como el fósil guía que define la zona junto a otros conodontes típicos de zonas más jóvenes; estos últimos son interpretados como retrabajados (Pohler y Orchard, 1990). También en el área de Nahanni, pero en la Formación Haywire, se encuentran conodontes de la Zona de Oepikodus evae (Pohler y Orchard, 1990).

La Zona de Oepikodus evae determinada en este trabajo se puede correlacionar con la Zona de Oepikodus evae registrada en parte de la Formación Green Point, Grupo Cow Head en la sección de St. Pauls Inlet y de Martin Point, Oeste de Terranova, Canadá (Johnston y Barnes, 1999).

En la sección de Huanghuachang, en el sur de China, se podría correlacionar la Zona de Oepikodus evae identificada aquí con la parte inferior de la Zona de Oepikodus evae que registran Wang et al. (2005).

En el archipiélago de Svalbard, Lehnert et al. (2013) estudian la bioestratigrafía del Grupo Oslobreen, en la Formación Valhallfonna, registrando la Zona de Oepikodus evae.

Stouge et al. (2016) analizan bloques de calizas que presentan evidencias de transporte, contenidas en la Formación Rosroe, Área de Lough Nafooey, oeste de Irlanda, reconociendo la Zona de Oepikodus evae.

En relación con los esquemas bioestratigráficos clásicos de la región baltoescandinava, esta biozona se puede correlacionar con la Zona de $O$. evae de Lindström (1971), y con la Zona de O. evae y la parte inferior de la Zona de Trapezognathus diprion de Bagnoli y Stouge (1997).

\subsection{Zona de Oepikodus intermedius}

Albanesi et al. (1998) definen esta biozona para la Formación San Juan, en la sección de Portezuelo Yanso; el límite inferior de la biozona coincidiría con la última aparición en el registro de Oepikodus evae. El límite superior estaría indicado por la primera aparición de Tripodus laevis Bradshaw. Esta unidad es una biozona de intervalo basada en la parte del 
rango de un taxón determinada por el de otros dos taxones y se corresponde al Floiano superior.

Observaciones en el área de estudio y discusión: la presencia de Oepikodus intermedius (Fig. 7), que da nombre a esta biozona, se registra en las muestras LG 8 y LG 12 y no se lo encuentra asociado con O. evae, ni con Tripodus laevis Bradshaw (Fig. 4). El límite inferior de la zona estaría indicado por la primera aparición en el registro de $O$. intermedius y se localizaría en el nivel LG 8. El límite superior se correspondería al nivel LG 16, en donde se registra por primera vez la especie Triangulodus brevibasis (Sergeeva), que en los registros de otras localidades de la Precordillera se extiende desde la parte media de la Zona de Baltoniodus triangularis-Tripodus laevis hasta la parte inferior de la siguiente biozona.

Cabe señalar, que la especie guía Oepikodus intermedius, no se registra en todo el intervalo, probablemente debido a algún tipo de condicionamiento ambiental. La especie Bergstroemognathus extensus Serpagli, que en la biozona anterior era dominante, desaparece, y Scolopodus krummi, también dominante, disminuye en abundancia, observándose solo elementos aislados. Albanesi (1998a) observa que en esta biozona se registra la última aparición de Tropodus australis (Serpagli) (Fig. 5), en coincidencia con el presente estudio donde se halla en un único nivel.

Correlación regional: en la Formación San Juan situada en Pachaco, sobre el margen del río San Juan, la fauna C de Serpagli (1974) presenta ejemplares de Oepikodus intermedius en ausencia de O. evae, Tripodus laevis o Baltoniodus triangularis, lo que permitiría correlacionarla con la Zona de Oepikodus intermedius identificada en este trabajo.

En el Bloque de San Rafael, Lehnert et al. (1998) correlacionan la parte superior de la Formación Ponón Trehué con la Zona de Oepikodus intermedius.

En la sección de Niquivil de la Precordillera Central de San Juan esta biozona se correlacionaría con la Zona de Asociación V (Oistodus aff. O. lanceolatus/ Juanognathus jaanussoni) de Lehnert (1995) y con la Zona de O. intermedius de Albanesi et al. (2006) reconocidas en la Formación San Juan.

En la quebrada de Talacasto, Precordillera Central de San Juan, Soria et al. (2013) recuperaron conodontes provenientes de grainstones y packstones biointraclásticos, que permitieron reconocer la Zona de Oepikodus intermedius en la Formación San Juan.

Carlorosi y Heredia (2013) analizan muestras obtenidas de la Formación Acoite, Cordillera
Oriental, noroeste de Argentina, y registran la Zona de Trapezognathus diprion. Además, proponen la Zona de Baltoniodus cf. B. triangularis por encima de la misma, las cuales se correlacionan con el tramo inferior y superior de la Zona de $O$. intermedius del esquema bioestratigráfico de Albanesi y Ortega (2016), respectivamente.

En la sección Laguna Verde, Sierra de Zenta, Cordillera Oriental, Noroeste de Argentina, Voldman et al. (2013) registran ejemplares de Baltoniodus cf. B. triangularis (Lindström), verificando la presencia de la zona homónima.

Correlación intercontinental: la Zona de Oepikodus intermedius reconocida en la Formación San Juan se podría correlacionar con la Zona de $O$. communis (Ethington y Repetski, 1984), y con el intervalo Cooperignathus aranda-Juanognathus jaanussoni de Ethington y Clark (1981) para la parte media de la Formación Wah Wah del Grupo Pogonip en Ibex, Utah. Además, esta unidad se podría correlacionar con la Zona de Oistodus multicorrugatusPeriodon flabellum del sur de China y con la Zona de Aurilobodus leptosomatus-Loxodus dissectus del norte de China (Wang, 1990).

En la región Báltica se puede correlacionar con la parte superior de la Zona de O. evae (Lindström, 1971) y con la Zona de Microzarkodina sp. A de Bagnoli y Stouge (1997) y en el archipiélago de Svalbard, Lehnert et al. (2013) estudian la bioestratigrafía del Grupo Oslobreen, Formación Valhallfonna, registrando la Zona de Oepikodus intermedius, que se correlacionaría con la Zona de $O$. intermedius y la base de la Zona de Baltoniodus triangularis-Tripodus laevis identificadas en el presente trabajo.

\subsection{Zona de Baltoniodus triangularis-Tripodus laevis}

Albanesi y Ortega (2016) definen esta biozona en la Formación San Juan a partir del registro identificado en una publicación preliminar (Della Costa y Albanesi, 2016); el límite inferior de la biozona estaría indicado por la primera aparición en el registro de Baltoniodus triangularis y de Tripodus laevis. El límite superior coincidiría con la primera aparición de Baltoniodus navis. Esta biozona de asociación; se corresponde desde el límite FloianoDapingiano hasta el Dapingiano inferior.

Observaciones en el área de estudio y discusión: la especie Tripodus laevis (Fig. 7), se registra en los niveles LG 28, LG 30 y LG 34 (Fig. 4). Sin embargo, 


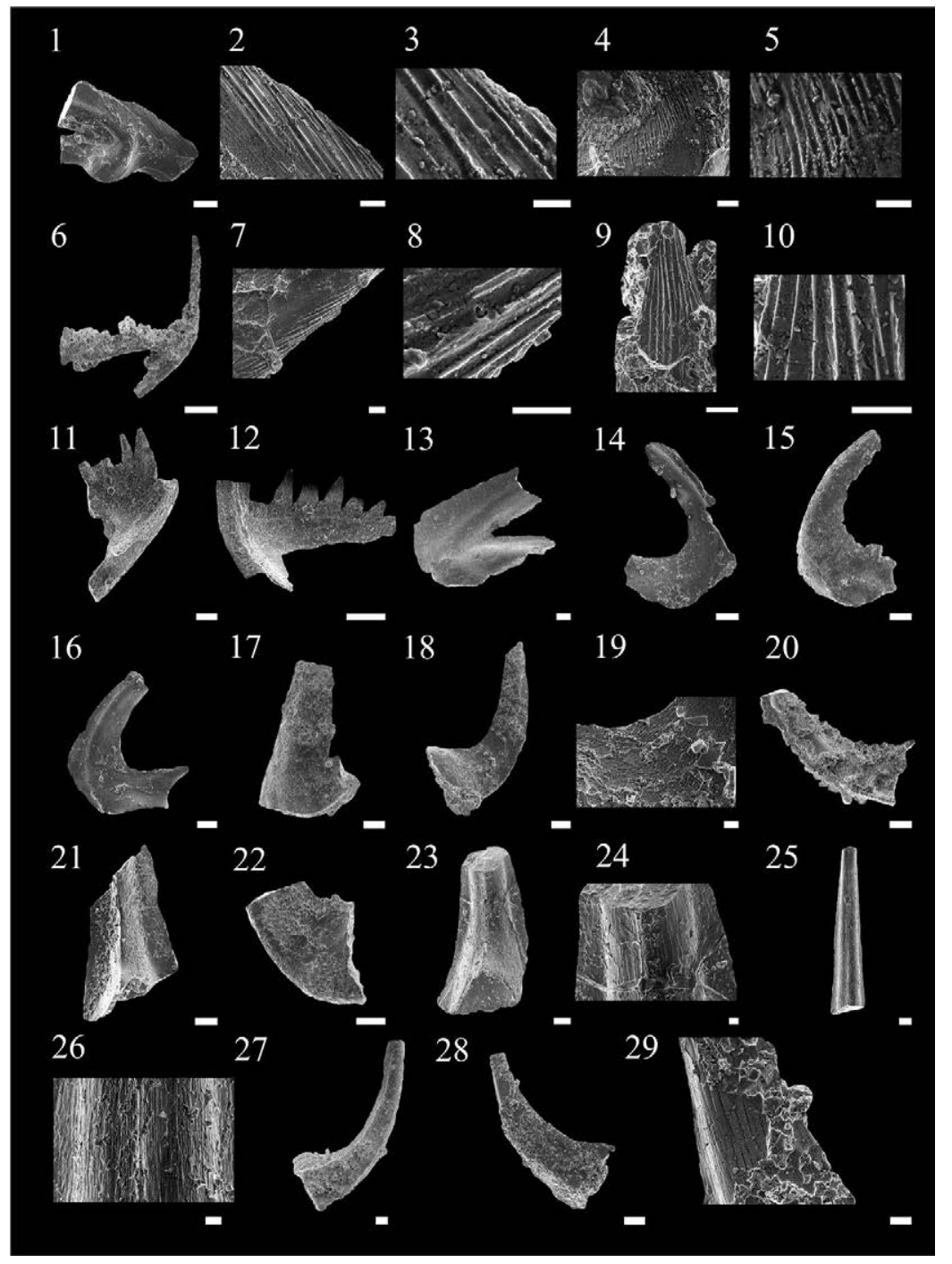

FIG. 7. 1-12. Oepikodus intermedius Serpagli; 1. M, LG 12, E.G. 50 mm, CORD-MP 56594; 2. detalle de estrías presentes en el margen anterior del proceso anterior, observables en el elemento de la figura 1, LG 12, E.G. $10 \mathrm{~mm}$; 3. estrías en el margen anterior del proceso anterior, observables en el elemento de la figura 1, LG 12, E.G. 5 mm; 4. estrías en la cúspide y margen superior de la base, observables en el elemento de la figura 1, LG 12, E.G. $10 \mathrm{~mm}$; 5. estrías en la cúspide y margen superior de la base, observables en el elemento de la figura 1, LG 12, E.G. 5 mm; 6. Sc, LG 10, E.G. 100 mm, CORD-MP 56595; 7. estrías en el margen anterior del proceso anterior, observables en el elemento de la figura 6, LG 10, E.G. 10 mm; 8. estrías en el margen anterior del proceso anterior, observables en el elemento de la figura 6, LG 10, E.G. 5 mm; 9. estrías en los dentículos, observables en el elemento de la figura 6, LG 10, E.G. $10 \mathrm{~mm}$; 10. estrías en los dentículos, observables en el elemento de la figura 6, LG 10, E.G. 5 mm; 11. Sd, LG 12, E.G. 50 mm, , CORD-MP 56596; 12. Pb, LG 10, E.G. 50 mm, CORD-MP 56597; 13-16. Paroistodus horridus primus Albanesi; 13. M, LG 42, E.G. 50 mm, CORD-MP 56598; 14. Sb, LG 42, E.G. 50 mm, CORD-MP 56599; 15. Sc, LG 42, E.G. 50 mm, CORD-MP 56600; 16. P, LG 42, E.G. 50 mm, CORD-MP 56601; 17-19. Paltodus? jemtlandicus Löfgren; 17. M, LG 30, E.G. 50 mm, CORD-MP 56602; 18. S, LG 34, E.G. 50 mm, CORD-MP 56603; 19. estrías en el margen posterior de la cúspide, observables en el elemento de la figura 18, LG 34, E.G. 10 mm; 20-22. Tripodus laevis Bradshaw; 20. Pb, LG 34, E.G. 50 mm, CORD-MP 56604; 21. Sa, LG 30, E.G. 50 mm, CORDMP 56605; 22. Sc, LG 28, E.G. 50 mm, CORD-MP 56606; 23-29. Triangulodus brevibasis (Sergeeva); 23. Sa, LG 16, E.G. $50 \mathrm{~mm}$, CORD-MP 56607; 24. estrías en la cúspide, observables en el elemento de la figura 23, LG 16, E.G. 10 mm; 25. Sa (cúspide del elemento de la imagen 23), LG 16, E.G. 50 mm, CORD-MP 56608; 26. estrías en la cúspide, observables en el elemento de la figura 25, LG 16, E.G. 10 mm; 27. Sc, LG 20, E.G. 50 mm, CORD-MP 56609; 28. Sd, LG 20, E.G. 50 mm, CORD-MP 56610; 29. estrías en la cúspide, observables en el elemento de la figura 28, LG 20, Escala gráfica. $10 \mathrm{~mm}$. 
el límite inferior de la zona se hallaría en el nivel LG 16, indicado por la primera aparición en el registro de Triangulodus brevibasis, que en los registros de otros sitios de la Precordillera se extiende desde la parte media de la Zona de Baltoniodus triangularisTripodus laevis hasta la parte inferior de la siguiente biozona, verificando que ese nivel ya se localizaría dentro de la Zona de B. triangularis-T. laevis.

El límite superior estaría por encima del nivel LG 34, ya que en este se halla la especie que da nombre a la biozona y las muestras localizadas por encima resultaron estériles.

La especie Tripodus laevis, que da nombre a la biozona, únicamente se registra en los niveles LG 28, LG 30 y LG 34, probablemente debido a algún sesgo paleoambiental o metodológico de laboratorio.

La especie T. laevis aparece asociada a Ansella jemtlandica, Drepanoistodus basiovalis, Juanognathus jaanussoni, Periodon flabellum (Lindström), Semiacontiodus potrerillensis Albanesi (Fig. 5) y Parapaltodus simplicissimus Stouge, los que poseen rangos bioestratigráficos que superan los límites de la biozona. En este intervalo se han registrado los únicos ejemplares de Triangulodus brevibasis, Protopanderodus leonardii Serpagli, Coelocerodontus sp., Stolodus stola (Lindström), Oistodus striolatus Serpagli, Anodontus longus Stouge y Bagnoli, así como los últimos registros de Cornuodus bergstroemi Serpagli y de Scolopodus krummi.

Las asociaciones registradas en la sección estudiada son más productivas en conodontes, en relación con otras secciones de la Precordillera, si bien no se registró una de las especies guía asociadas, i.e., B. triangularis.

Correlación regional: en la sección de Niquivil se correlaciona con la Zona de Asociación VI ("Parapanderodus" nogamii/Parapanderodus gracilis/ Ansella jemtlandica) de Lehnert (1995) y con la Zona de Tripodus laevis de Albanesi et al. (2006).

La Zona de B. triangularis-T. laevis de la Fm. San Juan es correlacionable con las asociaciones correspondientes a la Fauna D, registrada en el perfil de Pachaco (Serpagli, 1974), que en esta localidad como en la sección de Portezuelo Yanso y en la sección de Cerro Potrerillo (Albanesi et al., 1998), registran una escasa productividad de conodontes.

Carlorosi (2013) define la Zona de Baltoniodus triangularis para la Formación Alto del Cóndor, sierra de Los Colorados, Cordillera Oriental de Jujuy, la que se puede correlacionar con la Zona de
Baltoniodus triangularis-Tripodus laevis reconocida en este estudio.

Correlación intercontinental: la Zona de Baltoniodus triangularis-Tripodus laevis se correlacionaría, dentro de los esquemas bioestratigráficos baltoescandinavos, con las zonas de Baltoniodus? triangularis y Microzarkodina flabellum de Bagnoli y Stouge (1997), y con la Zona de Baltoniodus triangularis de Lindström (1971), Löfgren (1978) y Tolmacheva (2001). Lehnert et al. (2013) estudian la bioestratigrafía del Grupo Oslobreen, Formación Valhallfonna, del archipiélago de Svalbard, registrando la Zona de Oepikodus intermedius, cuya parte superior se correlacionaría con la base de la Zona de Baltoniodus triangularis-Tripodus laevis del presente trabajo; además registran, en parte de la Formación Valhahhfonna, la Zona de Periodon aff. flabellum, cuyo tramo inferior se correlacionaría con el tramo superior de la Zona de Baltoniodus triangularis-Tripodus laevis.

Ji y Barnes (1994) definen la Zona de Parapanderodus retractus, para ambientes de aguas someras, y la Zona de Pteracontiodus cryptodens, para ambientes de aguas profundas en la Formación Aguathuna, Grupo Saint George, Terranova, las que se correlacionarían con la Zona de Baltoniodus triangularis-Tripodus laevis reconocida en este estudio. Asimismo, se puede correlacionar con la Zona de Tripodus laevis registrada en parte de la Formación Green Point, Grupo Cow Head, en la sección de St. Pauls Inlet, y de Martin Point, Oeste de Terranova, Canadá (Johnston y Barnes, 1999).

A su vez, se corresponde con la Zona de Periodon hankensis de Stouge (2012), reconocida en parte de la Formación Shallow Bay, y de la Formación Green Point del Grupo Cow Head, oeste de Terranova, Canadá.

En la sección de Huanghuachang, China, se correlaciona con la Zona de Baltoniodus triangularis (Wang et al., 2003).

\subsection{Zona de Lenodus variabilis}

Albanesi et al. (1998) definen esta biozona en la Formación San Juan, sección de Portezuelo Yanso. El límite inferior de esta biozona coincidiría con la primera aparición de Periodon gladysae Albanesi, en los estratos cuspidales de la Formación San Juan, donde el límite superior permanece abierto dada la ausencia de registros. Esta unidad es una biozona de 
asociación que representa al Darriwiliano inferior alto a medio bajo.

En esta biozona, los autores reconocen dos subzonas; una inferior, denominada Subzona de Periodon gladysae (Subbiozona de intervalo basada en primeros registros), y una superior, denominada Subzona de Paroistodus horridus (Subbiozona de asociación).

El límite inferior de la Subzona de Periodon gladysae coincidiría con la aparición en el registro de la especie que le da nombre a la subzona, en tanto, el límite superior estaría indicado por el primer registro de Baltoniodus medius Löfgren. El límite inferior de la Subzona de Paroistodus horridus, está indicado por el último registro indicado y la primera aparición de Paroistodus horridus primus Albanesi y el registro de Baltoniodus medius. Si bien el límite superior no se pudo reconocer para la sección del cerro Potrerillo, estaría dado por la primera aparición de Yangtzeplacognathus crassus en otras localidades precordilleranas.

Observaciones en el área de estudio y discusión: la presencia de Lenodus sp. (Fig. 6) asociado con Fahraeusodus marathonensis (Bradshaw), Drepanodus reclinatus, Drepanoistodus costatus Abaimova, Histiodella minutiserrata (Mound), Histiodella sinuosa (Graves y Ellison), Paroistodus horridus primus, Periodon macrodentatus (Chen y Zhang) y Protopanderodus robustus (Hadding), permite identificar la Zona de Lenodus variabilis entre los niveles LG 41 y el nivel LG 42 (techo de la Formación San Juan) (Fig. 4).

El límite inferior se asignaría tentativamente sobre el nivel LG 41, con la primera aparición en este registro de Fahraeusodus marathonensis, asociado a Drepanodus reclinatus; sin embargo, como no se hallaron registros entre el nivel LG 34 y LG 41, probablemente dicho límite se encuentre debajo de este. El límite superior se asignaría tentativamente al techo de la Formación San Juan (nivel LG 42).

En la sección estudiada, se observa el primer registro en esta biozona de Fahraeusodus marathonensis, Scolopodus striatus (Lindström), Drepanoistodus costatus (Fig. 5), Drepanodus reclinatus, Histiodella minutiserrata, Histiodella sinuosa, Lenodus sp., Oistodus lanceolatus, Paroistodus horridus primus, Periodon macrodentatus, Periodon sp., Protopanderodus robustus y Spinodus spinatus (Hadding), y se extienden los registros de Drepanodus arcuatus, Parapaltodus simplicissimus, Paroistodus originalis (Sergeeva), Protopanderodus rectus (Lindström) y Rossodus barnesi Albanesi, cuyos rangos estratigráficos superan los límites de la biozona.

La presencia de Paroistodus horridus primus, permitiría reconocer la subzona superior o Subzona de Paroistodus horridus. Este resultado difiere con los resultados de trabajos previos (Ortega, 1987; Ottone et al., 1999; Ortega et al., 2007), donde se reportan asociaciones de conodontes típicas de la Zona de Yangzeplacognathus crassus para secciones situadas hacia el sur del cerro Viejo de Huaco. Esta diferencia en el registro de conodontes se debe a que el techo de la Formación San Juan presenta un diacronismo donde los estratos más jóvenes (Zona de Yangzeplacognathus crassus) se observan en las secciones situadas hacia el sur.

Correlación regional: Sarmiento (1985) es la primer autora en identificar la Zona de $L$. variabilis en la Precordillera argentina, en los afloramientos de las formaciones San Juan y Gualcamayo del flanco oriental de la sierra de Villicum.

En los estratos inferiores de la Formación Rinconada, Sarmiento et al. (1988) recuperan una asociación de especies características de la parte alta de la Zona de Lenodus variabilis. Posteriormente, Lehnert (1995) halla una asociación de conodontes de los mismos estratos asignable a la subzona superior o Subzona de Paroistodus horridus, entre los que se encuentran las especies Lenodus variabilis (Sergeeva) e Histiodella sinuosa.

La Zona de Lenodus variabilis es documentada por Albanesi et al. (1995) en los estratos basales de la Formación Yerba Loca en la sección de la puerta de Ancaucha, sobre la vertiente occidental del cerro Alto de Mayo en a Precordillera Occidental.

Albanesi et al. (1998) reevalúan las formas asignadas por Hünicken y Ortega (1987) a Eoplacognathus suecicus Bergström en la parte cuspidal de la Formación San Juan, en la sección de cerro Viejo de Huaco, asignándolas a Lenodus variabilis.

En el cerro La Chilca, se reconoce la Zona de Lenodus variabilis entre los 15 y $6 \mathrm{~m}$ debajo del techo de la Formación San Juan, Precordillera Central de San Juan (Heredia y Mestre, 2013). Estos autores y Carrera et al. (2013) proponen que la primera aparición en el registro de Paroistodus horridus ocurriría dentro de la Zona de Yangtzeplacognathus crassus. Posteriormente, Serra et al. (2017) realizan un rango compuesto de los conodontes presentes en las secciones de Las Chacritas, quebrada Las 
Aguaditas y cerro La Chilca mediante el programa CONOP9, proponiendo que la primera aparición de Paroistodus horridus ocurriría en la Zona de Lenodus variabilis, verificando la biozonación originalmente propuesta (Albanesi et al., 1998).

En la quebrada Las Aguaditas, córdon de Los Blanquitos, Precordillera de San Juan, Feltes et al. (2016) analizan muestras correspondientes a la parte superior de la Formación San Juan y reconocen la Zona de Lenodus variabilis con sus dos subzonas, entre los 24 y los $17 \mathrm{~m}$ por debajo del contacto con la Formación Las Aguaditas.

Correlación intercontinental: Lindström (1971) define la Zona de Amorphognathus variabilis (=Lenodus variabilis) en la secuencia de calizas condensadas del Escudo Báltico, correspondiente al Piso Kunda (BIII) de la Serie Oelandiana. Posteriormente, Löfgren (1978) observa que la extensión temporal de la especie guía se restringe al Kunda temprano, a partir de lo cual redefine las unidades.

Esta unidad bioestratigráfica se correlacionaría con la Zona de Histiodella sinuosa y parte de la Zona de Histiodella holodentata correspondiente a diferentes esquemas norteamericanos (Ethington y Clark, 1981; Sweet, 1984).

La Zona de Lenodus variabilis se correlacionaría con la Zona de Periodon macrodentatus de Stouge (2012), reconocida en parte de la Formación Shallow Bay y de la Formación Green Point del Grupo Cow Head, oeste de Terranova, Canadá.

En China, An (1987) registra faunas asignables a esta biozona en China meridional. Wang y Bergström (1995) reconocen dicha biozona en la parte más alta de la Formación Ningkuo en la sección de Huangnitang, Zhejiang, China.

Al analizar muestras de la Formación Rosroe, Killary Bay Little, oeste de Irlanda, Stouge et al. (2016) recuperan una asociación de conodontes que indicaría una correspondencia con la Zona de P. macrodentatus, que a su vez se correlacionaría con la Zona de Lenodus variabilis.

\section{Provincialismo de conodontes}

Los conodontes ordovícicos exhibieron un marcado provincialismo, según fuera identificado originalmente por Sweet et al. (1971), Bergström (1971), Barnes et al. (1973), entre otros autores, que condujeron originalmentea la definición de dos provincias; i.e., la Provincia Norteamericana Midcontinent (PNAM) representada por faunas de aguas cálidas en la región ecuatorial y la Provincia Nordatlántica (PNA), distinguida por faunas de aguas frías en regiones de altas latitudes. Las provincias fueron definidas principalmente sobre la base de datos obtenidos de distintas regiones de Norteamérica y Europa, respectivamente (Bergström, 1973). La profundidad y la temperatura del agua habrían intervenido como factores físicos importantes en la diferenciación de las dos provincias (Bergström y Sweet, 1966).

En ámbitos de bajas latitudes, el límite entre las dos provincias habría coincidido con la separación entre el mar somero (plataforma) y el mar abierto (talud), donde el límite plataforma-talud produce una fuerte caída de la temperatura sobre el margen de la plataforma, tal como acontece en regiones tropicales como al este de Laurentia (Ethington et $a l ., 1995)$. Este cambio brusco habría sido menos prominente hacia altas latitudes, siendo más difícil diferenciar las faunas de aguas someras de las de aguas profundas. De esta manera, las faunas de plataforma en las regiones tropicales podrían asignarse a la Provincia Norteamericana Midcontinent, en tanto que las faunas del borde de la plataforma y talud de dichas regiones, como así también las faunas de altas latitudes (plataforma y talud) podrían atribuirse a la Provincia Nordatlántica (Pohler y Barnes, 1990; Dubinina, 2000).

Las faunas de aguas profundas del océano Iapetus estuvieron dominadas por especies cosmopolitas (Zhen y Percival, 2003), en tanto, las faunas de mar somero contuvieron numerosos taxones endémicos, principalmente en la Provincia Norteamericana Midcontinent.

A partir de esta división, se reconocieron faunas con afinidad a la Provincia Norteamericana Midcontinent en las regiones tropicales y someras de Laurentia, Siberia, centro-oeste de Australia, China norte y en algunos arcos de islas; por otro lado, se reconocieron faunas con afinidad a la Provincia Nordatlántica en las regiones de aguas templado-frías de Báltica, Kazakhstan, este de Australia, sur de China, Polonia, y otras regiones de menor extensión.

Otra división es la que propone Rasmussen (1998) mediante datos correspondientes a las zonas de Lenodus variabilis y de Histiodella holodentata de los esquemas bioestratigráficos de Baltoescandinavia y de Laurentia, respectivamente. El autor observa que la cercana similitud de las faunas del este de Laurentia y Baltoescandinavia se debería a la coexistencia 
de especies pandémicas, por lo que propone que se considere la totalidad de Laurentia en el Reino Midcontinent y a Báltica en el Reino Atlántico.

El modelo más reciente fue propuesto por Zhen y Percival (2003), quienes distinguieron dos reinos vinculados a la profundidad: el Reino de Mar Somero que abarcaría las faunas de la plataforma (menor a $200 \mathrm{~m}$ de profundidad) y el Reino de Mar Abierto que comprendería las faunas del talud (mayor a $200 \mathrm{~m}$ de profundidad). En cada reino reconocieron tres dominios controlados por la temperatura: los Dominios Tropical, Templado y Frío. En el Reino de Mar Somero, el Dominio Tropical se caracterizó por poseer faunas más diversas y un mayor porcentaje de endemismo en relación a los otros; el Dominio Templado con una diversidad moderada y el Dominio Frío con una baja diversidad y una alta abundancia. Dentro del Dominio Tropical, Zhen y Percival (2003) reconocieron las provincias Lauréntica, Australiana y China Norte; para el Dominio Templado, las provincias China Sur y de la Precordillera Argentina y en el Dominio Frío, a la Provincia Baltoescandinava. De acuerdo con este modelo, que se sigue en el presente trabajo, la PNAM correspondería a una particular provincia del Dominio Tropical del Reino de Mar Somero, y la PNA sería una provincia del Dominio Frío del Reino de Mar Abierto.

\subsection{Antecedentes sobre paleobiogeografía de conodontes en la Precordillera para el intervalo considerado en este estudio}

El reconocimiento de una unidad paleobiogeográfica de conodontes con características propias, la Provincia de Precordillera, fue propuesto originalmente por Serpagli (1974) para un intervalo similar al del presente estudio. Lehnert (1995), a partir del análisis de la macrofauna presente en diferentes secciones de la Precordillera, llega a una conclusión similar a Serpagli (1974). Bagnoli y Stouge (1991) evalúan las proporciones de faunas de distintos paleocontinentes presentes en la Precordillera, reconociendo la Provincia Precordillerana dentro del Reino Atlántico de agua templado-fría para el Ordovícico Temprano.

Posteriormente, luego de un análisis detallado de la fauna recuperada en las secciones del cerro Potrerillo y Portezuelo Yanso, Albanesi (1998b) distingue a la Precordillera como una provincia de aguas templadas del Reino Nordatlántico para el Floiano. Por otra parte, Christiansen y Stouge (1999) reconocen a la Provincia Precordillerana, separada de las provincias Midcontinent y Nordatlántica.

Albanesi y Bergström $(2004,2010)$ comparan la diversidad faunística de conodontes registrados en la Precordillera para el intervalo TremadocianoDarriwiliano, con la de distintas localidades del mundo, que durante el Ordovícico formaban parte de Laurentia, Báltica y Gondwana. Sobre el intervalo que incluiría las zonas de Oepikodus evae, Oepikodus intermedius, Baltoniodus triangularis-Tripodus laevis, Baltoniodus navis, Microzarkodina parva y Lenodus variabilis, los autores observan que la diversidad de conodontes posee afinidad con las faunas de Laurentia del área de Marathon, situada en Texas, y que esta no disminuye a través de ese intervalo de tiempo; por otra parte, detectan una gran cantidad de especies de distribución cosmopolita.

\subsection{Análisis de la fauna recuperada}

Al observar la diversidad de géneros en todo el intervalo estudiado (Fig. 8A), se advierte que predominan los géneros con distribución cosmopolita $(74,19 \%)$, pero se reconocen también taxones compartidos únicamente con la Provincia Norteamericana Midcontinent (PNAM) (19,35\%) y con la Provincia Nordatlántica (PNA) $(6,45 \%)$. En el gráfico de frecuencias relativas de especies (Fig. 8B), se observa que la distribución es más homogénea entre especies con distribución cosmopolita $(52,08 \%)$ y aquellas compartidas solo con la PNAM $(35,42 \%)$, y se encuentra un menor porcentaje de especies compartidas con la PNA $(8,33 \%)$ y de la Precordillera (4,16\%). Esta diferencia es debido a que el endemismo se revela apropiadamente analizando la distribución a nivel de especie (Ludvigsen et al., 1986; Maciel-Mata et al., 2015); por otra parte, la gran cantidad de taxones con distribución cosmopolita sería coincidente con la hipótesis del desprendimiento del Terreno de Cuyania (que incluiría a la Precordillera) y su posterior deriva a través del océano Iapetus, el que se habría caracterizado por presentar este tipo de taxones, para este intervalo temporal (Albanesi y Bergström, 2010); a su vez, incluyendo taxones característicos de la PNAM y PNA por el corredor oceánico que la conectaría a Laurentia y a Báltica.

$\mathrm{Al}$ analizar la distribución de los géneros en cada piso estudiado, se desprende que en el Floiano (Fig. 9,A) se detecta una amplia participación de taxones con distribución cosmopolita y, en menor proporción, 

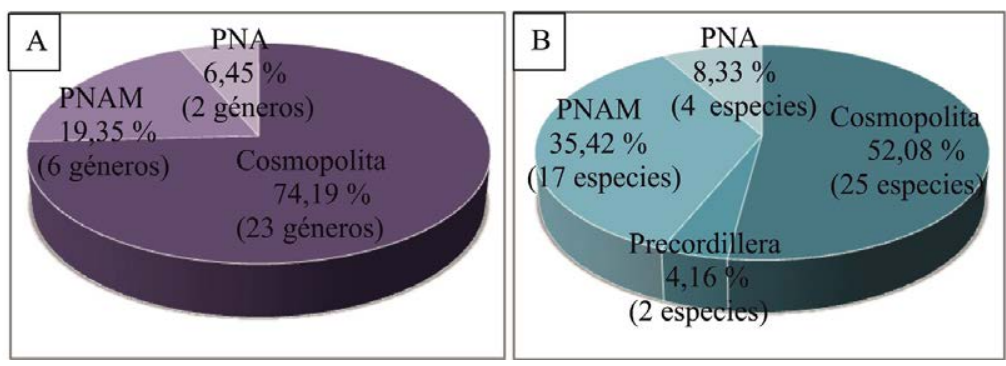

FIG. 8. A. Frecuencia relativa de géneros endémicos de la Precordillera, compartidos únicamente con la Provincia Norteamericana Midcontinent (PNAM), o con la Provincia Nord-atlántica (PNA), y de distribución cosmopolita; B. Frecuencia relativa de especies endémicas de la Precordillera, compartidas únicamente con la Provincia Norteamericana Midcontinent (PNAM), o con la Provincia Nord-atlántica (PNA), y de distribución cosmopolita.

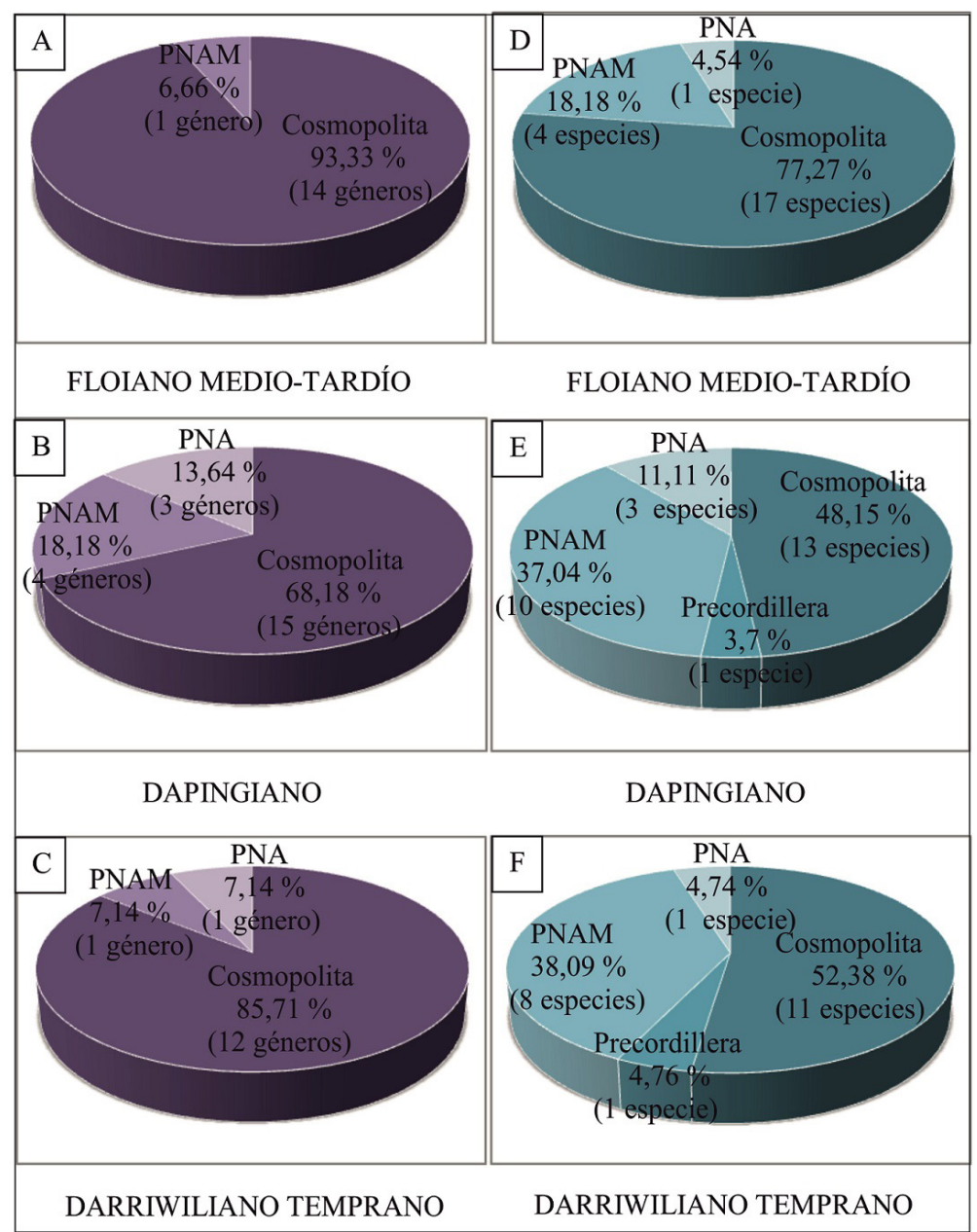

FIG. 9. A-C. Frecuencia relativa de géneros endémicos de la Precordillera, compartidos únicamente con la Provincia Norteamericana Midcontinent (PNAM), o con la Provincia Nord-atlántica (PNA), y de distribución cosmopolita. A. Durante el Floiano mediotardío; B. Durante el Dapingiano; C. Durante el Darriwiliano temprano; D-F. Frecuencia relativa de especies endémicas de la Precordillera, compartidas únicamente con la Provincia Norteamericana Midcontinent (PNAM), o con la Provincia Nordatlántica (PNA), y de distribución cosmopolita; D. Durante el Floiano medio-tardío; E. Durante el Dapingiano; F. durante el Darriwiliano temprano. 
compartidos solo con la Provincia Norteamericana Midcontinent. Durante el Dapingiano, y el Darriwiliano temprano (Fig. 9B y C), aumenta la proporción de géneros típicos de la PNAM y hacen su aparición géneros típicos de la Provincia Nordatlántica, con un porcentaje elevado de géneros que presentan distribución cosmopolita.

Para el Floiano medio-tardío se detectan especies (Fig. 9D) afines a la Provincia Norteamericana Midcontinent (PNAM), afines a la Provincia Nordatlántica (PNA) y de distribución cosmopolita principalmente, sin registrarse especies propias de la Precordillera. Durante el Dapingiano (Fig. 9E), aparecen en el registro especies endémicas de la Precordillera, apreciándose un aumento de la cantidad y porcentaje de especies de afinidad con la PNAM, de afinidad a la PNA, y una disminución de las especies de distribución cosmopolita. A través del Darriwiliano temprano (Fig. 9F), la cantidad y porcentaje de especies se mantiene similar, con la excepción de que las especies típicas de la PNA disminuyen su cantidad y porcentaje levemente. A través de todo el intervalo analizado, la cantidad y porcentaje de géneros y especies características de la PNAM es mayor a la de la PNA.

En este análisis se reconoce que la diversidad genérica (33 géneros) y específica (55 especies) de conodontes es elevada, y la abundancia genérica es baja por piso estudiado y a través de todo el intervalo analizado (Figs. 10 y 11; Tabla 1), tal como

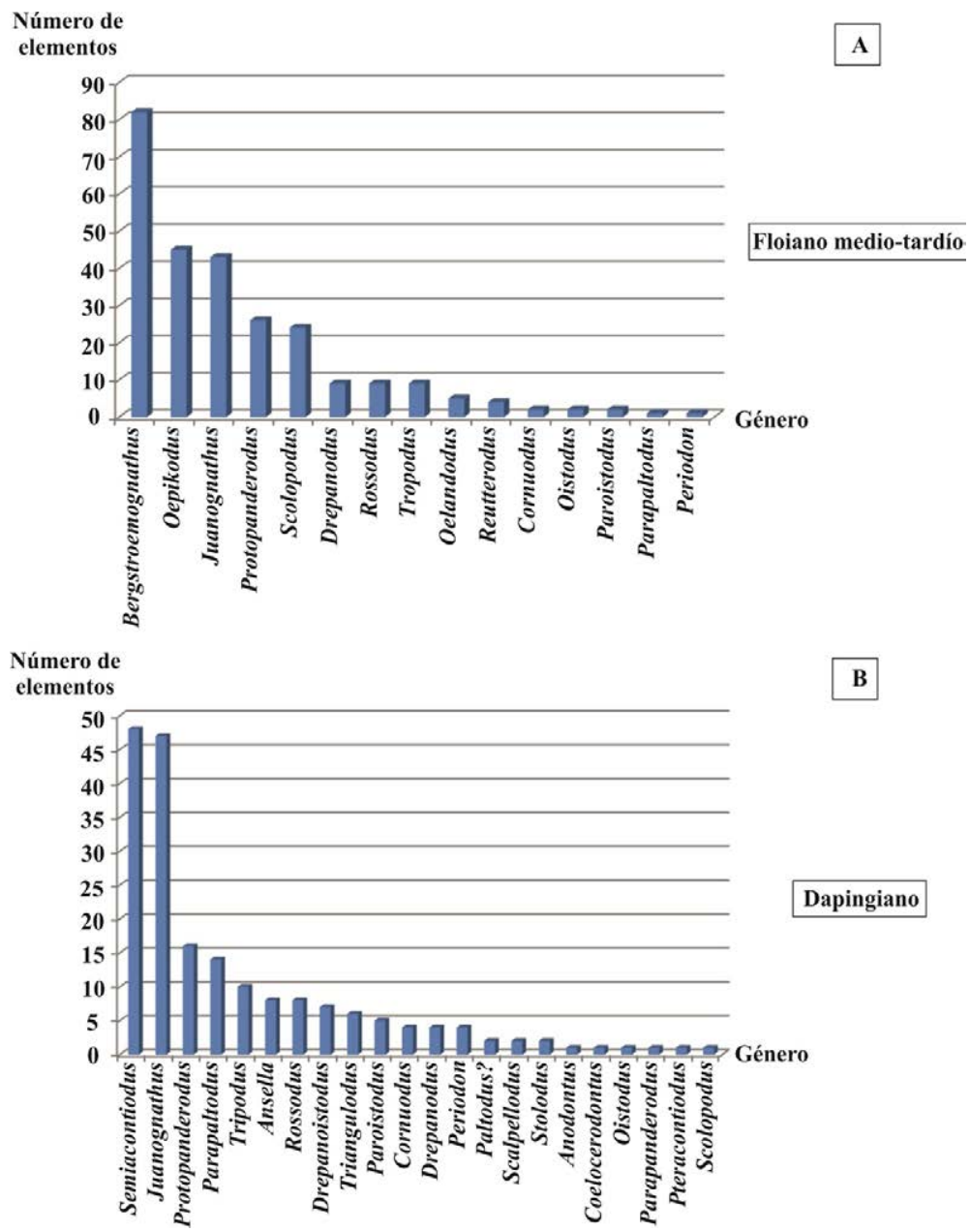

FIG. 10. Frecuencia absoluta de elementos por géneros de conodontes determinados en este estudio, para el intervalo Floiano mediotardío (A) y para el Dapingiano (B). 

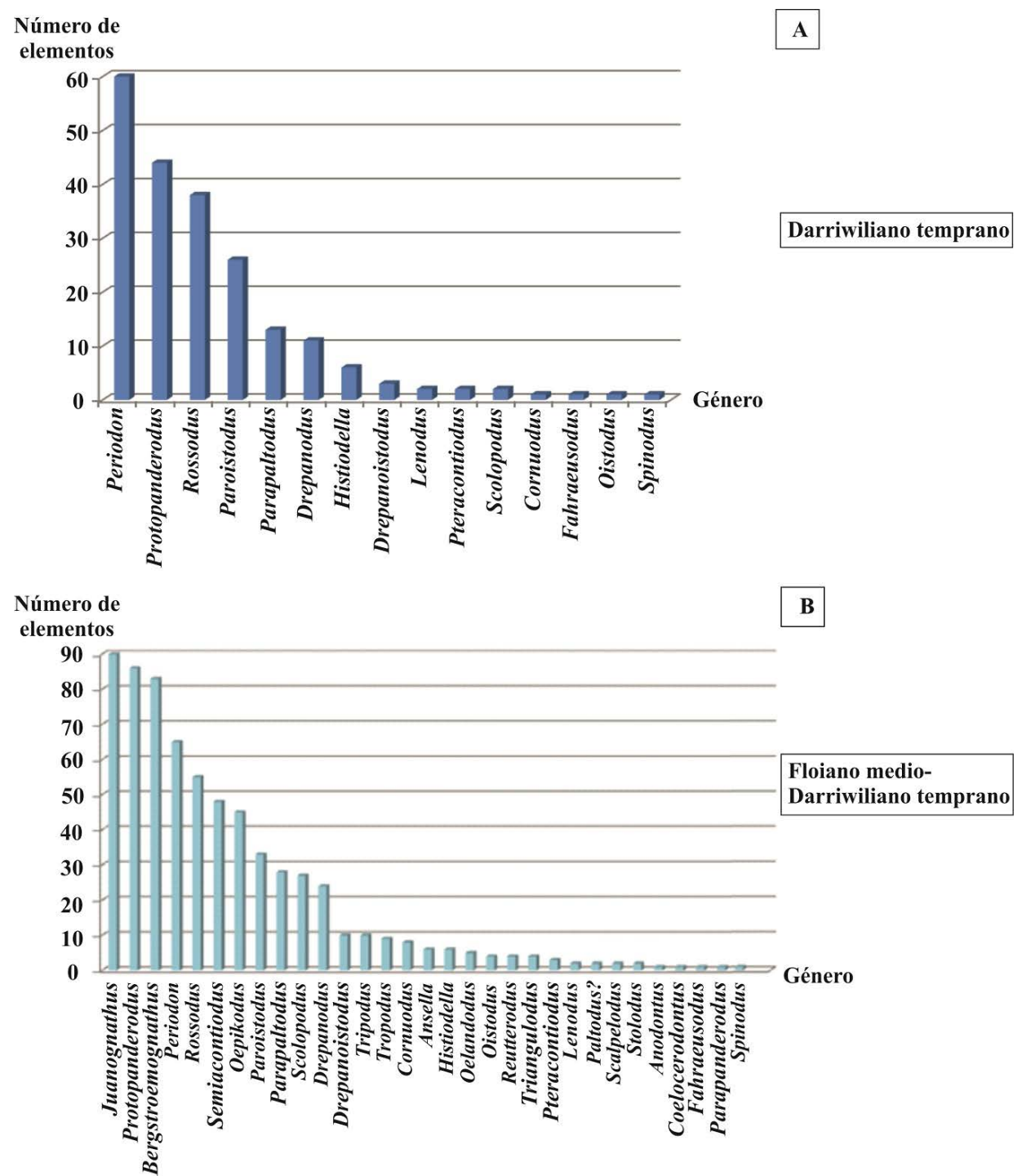

FIG. 11. Frecuencia absoluta de elementos por géneros de conodontes determinados en este estudio para el Darriwiliano temprano (A) y para todo el intervalo estudiado; B. Floiano medio-Darriwiliano temprano.

presentan aquellas áreas situadas en aguas cálidas; en tanto que la asociación de conodontes posee un alto porcentaje de taxones de distribución cosmopolita como registran Albanesi y Bergström $(2004,2010)$. Sin embargo, son las especies características en común las que definen la afinidad faunística, por lo que al observar un porcentaje en común, a nivel de especie, de entre $18 \%$ y $38 \%$ únicamente con la PNAM y de entre $4 \%$ y $11 \%$ únicamente con la PNA (Fig. 9D, E, F), se puede estimar una mayor afinidad con la PNAM, (que también presentaría un gran porcentaje de taxones cosmopolitas), y una menor con la PNA, indicando una mayor cercanía a Laurentia que a Báltica, lo que sería coincidente con la hipótesis de deriva del Terreno de Cuyania como se explicó previamente. No obstante, no se observa una variación de la cantidad de taxones en común con respecto a la PNAM, ni a la PNA, lo que indicaría una estabilidad faunística durante el intervalo temporal analizado.

La asociación faunística estudiada sería similar a la observada en la Formación San Juan para otros 
TABLA 1. FRECUENCIA ABSOLUTA DE ESPECIES DE CONODONTES DETERMINADAS EN ESTE TRABAJO (FORMACION SAN JUAN, FLOIANO MEDIO-DARRIWILIANO INFERIOR).

\begin{tabular}{|c|c|c|c|c|c|c|c|c|c|c|c|c|c|c|c|c|c|c|c|c|c|c|c|c|c|c|c|}
\hline Especies/Muestras & LG 0 & LG 1 & LG 2 & LG 4 & LG 6 & LG 8 & LG 10 & \begin{tabular}{|l|} 
LG 12 \\
\end{tabular} & \begin{tabular}{|l|l|} 
LG 14 \\
\end{tabular} & LG 14,1 & \begin{tabular}{|l|} 
LG 16 \\
\end{tabular} & LG 18 & LG 20 & LG 22 & LG 24 & LG 28 & LG 30 & LG 32 & LG 34 & LG 36 & \begin{tabular}{|l|} 
LG 38 \\
\end{tabular} & LG 39,9 & LG 40,1 & LG 41 & \begin{tabular}{|l|} 
LG 41,1 \\
\end{tabular} & LG 42 & Total \\
\hline Anodontus longus & & & & & & & & & & & & & & & 1 & & & & & & & & & & & & 1 \\
\hline Ansella jemtlandica & & & & & & & & & & & & & 1 & & & 1 & 4 & & 2 & & & & & & & & 8 \\
\hline Bergstroemognathus extensus & & & 25 & 11 & 46 & & & & & & & & & & & & & & & & & & & & & & 82 \\
\hline Coelocerodontus sp. & & & & & & & & & & & & & & & 1 & & & & & & & & & & & & 1 \\
\hline Cornuodus bergstroemi & & & & & 1 & & & 1 & & & & & & & & 1 & & & & & & & & & & & 3 \\
\hline Cornuodus longibasis & & & & & & & & & & & & & & & & & 1 & & 2 & & & & & 1 & & & 4 \\
\hline Drepanodus arcuatus & & & & & 4 & 1 & 1 & 3 & & & & & & & 1 & & 3 & & & & & & & & & 3 & 16 \\
\hline Drepanodus reclinatus & & & & & & & & & & & & & & & & & & & & & & & & & & 8 & 8 \\
\hline Drepanoistodus basiovalis & & & & & & & & & & & & 1 & & & & 5 & & & 1 & & & & & & & & 7 \\
\hline Drepanoistodus costatus & & & & & & & & & & & & & & & & & & & & & & & & 1 & & 1 & 2 \\
\hline Drepanoistodus sp. & & & & & & & & & & & & & & & & & & & & & & & & & & 1 & 1 \\
\hline Fahraeusodus marathonensis & & & & & & & & & & & & & & & & & & & & & & & & 1 & & & 1 \\
\hline Histiodella minutiserrata & & & & & & & & & & & & & & & & & & & & & & & & & & 1 & 1 \\
\hline Histiodella sinuosa & & & & & & & & & & & & & & & & & & & & & & & & & & 5 & 5 \\
\hline Juanognathus jaanussoni & & & & & 2 & 1 & 1 & 4 & & 1 & & & 6 & & 30 & 7 & 2 & & & & & & & & & & 54 \\
\hline Juanognathus serratus & & & & & & & & & & & & & & & 1 & 1 & & & & & & & & & & & 2 \\
\hline Juanognathus sp. & & & & & & & 1 & & & & & & & & & & & & & & & & & & & & 1 \\
\hline Juanognathus variabilis & & & 7 & 5 & 19 & 2 & & & & & & & & & & & & & & & & & & & & & 33 \\
\hline Lenodus sp. & & & & & & & & & & & & & & & & & & & & & & & & & & 2 & 2 \\
\hline Oelandodus costatus & & & & & 3 & & & & & & & & & & & & & & & & & & & & & & 3 \\
\hline Oelandodus elongatus & & & & & 2 & & & & & & & & & & & & & & & & & & & & & & 2 \\
\hline Oepikodus evae & & & 4 & 4 & 29 & & & & & & & & & & & & & & & & & & & & & & 37 \\
\hline Oepikodus intemedius & & & & & & 1 & 3 & 4 & & & & & & & & & & & & & & & & & & & 8 \\
\hline Oistodus lanceolatus & & & & & & & & & & & & & & & & & & & & & & & & & & 1 & 1 \\
\hline Oistodus sp. & & & & & 2 & & & & & & & & & & & & & & & & & & & & & & 2 \\
\hline Oistodus striolatus & & & & & & & & & & & & & & & 1 & & & & & & & & & & & & 1 \\
\hline Paltodus? jemtlandicus & & & & & & & & & & & & & & & & & 1 & & 1 & & & & & & & & 2 \\
\hline Parapaltodus simplicissimus & & & & & 1 & & & & & & & & 1 & & 10 & 1 & 2 & & & & & & & 2 & & 11 & 28 \\
\hline Parapanderodus paracornuformis & & & & & & & & & & & & & & & & & 1 & & & & & & & & & & 1 \\
\hline Paroistodus horridus primus & & & & & & & & & & & & & & & & & & & & & & & & & & 17 & 17 \\
\hline Paroistodus originalis & & & & & & & & & & & & 1 & & & 2 & 1 & & & 1 & & & & & 3 & & 6 & 14 \\
\hline Paroistodus parallelus & & & 1 & & & & & & & & & & & & & & & & & & & & & & & & 1 \\
\hline Paroistodus cf. $P$. proteus & & & & & 1 & & & & & & & & & & & & & & & & & & & & & & 1 \\
\hline Periodon flabellum & & & & & & & & 1 & & & & 1 & 2 & & & & 1 & & & & & & & 3 & & & 8 \\
\hline Periodon macrodentatus & & & & & & & & & & & & & & & & & & & & & & & & & & 56 & 56 \\
\hline Periodon sp. & & & & & & & & & & & & & & & & & & & & & & & & & & 1 & 1 \\
\hline Protopanderodus elongatus & & & & & & & & & & & & & & & & 1 & & & 1 & & & & & & & & 2 \\
\hline Protopanderodus gradatus & & & & & & 6 & 3 & & & & & & & 2 & 7 & & 2 & & & & & & & & & 30 & 50 \\
\hline Protopanderodus leonardii & & & & & & & & & & & & & 1 & & & & & & & & & & & & & & 1 \\
\hline Protopanderodus rectus & & & & & 14 & 1 & 2 & & & & & & & & & 1 & & & 1 & & & & & & & 1 & 20 \\
\hline Protopanderodus robustus & & & & & & & & & & & & & & & & & & & & & & & & & & 13 & 13 \\
\hline Pteracontiodus cryptodens & & & & & & & & & & & & & 1 & & & & & & & & & & & & & 2 & 3 \\
\hline Reutterodus andinus & & & 1 & & 3 & & & & & & & & & & & & & & & & & & & & & & 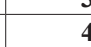 \\
\hline Rossodus barnesi & & & 1 & & 7 & & & 1 & & & & & 4 & 1 & 1 & 2 & & & & & & & & 5 & & 33 & 55 \\
\hline Scalpellodus gracilis & & & & & & & & & & & & & & & & 2 & & & & & & & & & & & 2 \\
\hline Scolopodus krummi & & & & 1 & 23 & & & & & & & 1 & & & & & & & & & & & & & & & 25 \\
\hline Scolopodus striatus & & & & & & & & & & & & & & & & & & & & & & & & 2 & & & 2 \\
\hline Semiacontiodus potrerillensis & & & & & & & & & & & & 2 & 2 & 1 & 3 & 3 & 22 & & 14 & & & & & & & & 47 \\
\hline Semiacontiodus sp. & & & & & & & & & & & & & & & 1 & & & & & & & & & & & & 1 \\
\hline Spinodus spinatus & & & & & & & & & & & & & & & & & & & & & & & & & & 1 & 1 \\
\hline Stolodus stola & & & & & & & & & & & & & & & 2 & & & & & & & & & & & & 2 \\
\hline Triangulodus brevibasis & & & & & & & & & & & 1 & & 3 & & & 2 & & & & & & & & & & & 6 \\
\hline Tripodus laevis & & & & & & & & & & & & & & & & 2 & 6 & & 2 & & & & & & & & 10 \\
\hline Tropodus australis & & & & & & & & 2 & & & & & & & & & & & & & & & & & & & 2 \\
\hline Tropodus sweeti & & & & & 7 & & & & & & & & & & & & & & & & & & & & & & 7 \\
\hline Total de elementos por muestra & $\mathbf{0}$ & $\mathbf{0}$ & 39 & 21 & 164 & 12 & 11 & 16 & 0 & 1 & 1 & 6 & 21 & 4 & 61 & 30 & 45 & $\mathbf{0}$ & 25 & 0 & 0 & $\mathbf{0}$ & 0 & 18 & 0 & 193 & 668 \\
\hline Cantidad de material procesado (gr) & 500 & 500 & 500 & 500 & 500 & 500 & 500 & 500 & 500 & 500 & 500 & 500 & 500 & 500 & 500 & 500 & 500 & 500 & 500 & 500 & 500 & 1.000 & 500 & 1.000 & 500 & 1.000 & 14.500 \\
\hline Material no disuelto (gr) & 0 & 0 & 0 & 0 & 0 & $\mathbf{0}$ & $\mathbf{0}$ & 0 & 0 & 0 & 0 & 0 & 0 & 5 & 5 & 0 & 50 & 40 & 0 & 5 & 20 & 200 & 160 & 290 & 20 & 5 & 800 \\
\hline Material disuelto (gr) & 500 & 500 & 500 & 500 & 500 & 500 & 500 & 500 & 500 & 500 & 500 & 500 & 500 & 495 & 495 & 500 & $\begin{array}{r}450 \\
\end{array}$ & 460 & 500 & 495 & 480 & 800 & 340 & 710 & 480 & 995 & 13.700 \\
\hline
\end{tabular}


sectores de la Precordillera Central, considerando el mismo intervalo temporal, como se observa en la sección de Pachaco (Serpagli, 1974), Niquivil (Lehnert, 1993,1995; Albanesi et al. 2006), cerro Viejo de Huaco (Mestre, 2008; Mango et al. 2016), Portezuelo Yanso y cerro Potrerillo (Albanesi et al., 1998), sierra de Villicum (Sarmiento, 1985), cerro La Chilca (Heredia y Mestre, 2013, Serra et al., 2017) y la quebrada Las Aguaditas en el cordón de Los Blanquitos (Feltes et al., 2016). Además, tendría muchos taxones en común con áreas que se corresponderían a la PNAM como con el este y norte de Groenlandia (Smith, 1991), Canadá (Pohler y Orchard, 1990; Stouge, 2012), Terranova (Johnston y Barnes, 1999; Stouge, 2012), Estados Unidos (Ethington y Clark, 1981; Ethington y Repetski, 1984; Sweet, 1984) y el norte de China (Wang, 1990). Y una menor cantidad de taxones en común con áreas que se corresponderían a la PNA como el sur de China (Wang et al., 2005; Wang, 1990) y Suecia (e.g., Bagnoli y Stouge, 1997).

El presente estudio analiza los cambios faunísticos de conodontes en el paleoambiente de rampa carbonática; posiblemente por este motivo no se detecta una transición faunística desde el Dominio Tropical (Ordovícico Temprano) al Dominio Templado (Ordovícico Medio) como mencionan Albanesi y Ortega (2016) (cf. Albanesi y Bergström, 2004, 2010), en la síntesis bioestratigráfica que abarca a las distintas secciones estudiadas de la Precordillera argentina, sino que la asociación faunística registrada en este trabajo sería típica del Dominio Tropical (Zhen y Percival, 2003). Sin embargo, se reconoce la Precordillera como una provincia con características propias como identifican diversos autores (Serpagli, 1974; Lehnert, 1995; Bagnoli y Stouge, 1991; Albanesi, 1998b; Christiansen y Stouge, 1999).

\section{Conclusiones}

Los conodontes recuperados en el tramo mediosuperior de la Formación San Juan permiten reconocer las zonas de Oepikodus evae, Oepikodus intermedius, Baltoniodus triangularis-Tripodus laevis y Lenodus variabilis, que cubren el intervalo cronoestratigráfico Floiano medio a Darriwiliano inferior.

Las zonas de Baltoniodus navis y de Microzarkodina parva no se registraron a causa de la deficiente información sobre conodontes en el intervalo correspondiente.
En la Zona de Baltoniodus triangularis-Tripodus laevis, se observa una alta frecuencia de conodontes, a diferencia de otras localidades de la Precordillera, revelándose un perfil apto para diversos análisis faunísticos sobre esta unidad bioestratigráfica.

El análisis de la riqueza genérica y específica por intervalos temporales sucesivos presenta un gran porcentaje de taxones con distribución cosmopolita, una amplia participación de formas típicas de la Provincia Norteamericana Midcontinent (entre 18 y $38 \%$ a nivel de especie), y una discreta participación de taxones de la Provincia Nordatlántica, lo que indicaría una mayor afinidad faunística a la PNAM que a la PNA durante todo el intervalo analizado. El gran porcentaje de taxones con distribución cosmopolita sería coincidente con la hipótesis del desprendimiento del Terreno de Cuyania (que incluiría a la actual Precordillera) y su posterior deriva a través del océano Iapetus (el que se habría caracterizado por presentar este tipo de taxones) para este intervalo temporal; además, presenta taxones característicos de la PNAM y PNA debido a un fluido intercambio faunístico que posibilitaría el corredor oceánico que la vincularía a Laurentia y a Báltica. Por otra parte, se reconoce la Precordillera como una provincia con características propias, a partir de un número de formas endémicas, tal como la identifican diversos autores.

Se observa una alta diversidad de géneros y de especies, y escasa abundancia de especímenes a nivel genérico, similar a aquellas zonas que habrían correspondido a ambientes marinos de aguas cálidas, durante el Floiano medio a Darriwiliano temprano.

Los conodontes analizados en este estudio presentan características de preservación superficial que revelan efectos de procesos tafonómicos, $\mathrm{y}$ un CAI 2-2,5, que indicaría paleotemperaturas de soterramiento de entre $60^{\circ}$ y $155^{\circ} \mathrm{C}$.

\section{Agradecimientos}

Los autores desean agradecer a los evaluadores y a la Dra. A. Concheyro por sus valiosas recomendaciones; a la Dra. C. Marchi y al Centro de Microscopía Avanzada (CMA) de la Facultad de Ciencias Exactas y Naturales, UBA, situado en el Pabellón I. Asimismo, agradecen al CONICET y a la Universidad Nacional de Córdoba, por el apoyo permanente al estudio de conodontes.

El contenido de esta contribución corresponde a una parte de la Tesis Doctoral en Ciencias Geológicas del primer autor. 


\section{Bibliografía}

Albanesi, G.L. 1998a. Taxonomía de conodontes de las secuencias ordovícicas del cerro Potrerillo, Precordillera Central de San Juan, R. Argentina. Actas de la Academia Nacional de Ciencias, Tomo 12: 102-253. Córdoba.

Albanesi, G.L. 1998b. Biofacies de conodontes de las secuencias ordovícicas del cerro Potrerillo, Precordillera Central de San Juan, R. Argentina. Actas de la Academia Nacional de Ciencias, Tomo 12: 75-98. Córdoba.

Albanesi, G.L.; Astini, R.A. 2000. Nueva fauna de conodontes de la Formación Suri (Ordovícico Inferior-Medio), Sistema de Famatina, Argentina. In Reunión anual de comunicaciones de la Asociación Paleontológica Argentina. Resumen, Ameghiniana 37 (4): p. 68. Mar del Plata.

Albanesi, G.L.; Ortega, G. 2002. Advances on conodontgraptolite biostratigraphy of the Ordovician System in Argentina. In Aspects on the Ordovician System in Argentina (Aceñolaza, F.G.; editor). Serie Correlación Geológica, No. 16, Instituto Superior de Correlación Geológica: 143-165. Tucumán.

Albanesi, G.L.; Bergström, S.M. 2004. Conodonts: Lower to Middle Ordovician Record. In The Great Ordovician Biodiversification Event (Webby, B.D.; Paris, F.; Droser, M.L.; Percival, I.G.; editores). Columbia University Press : 312-336. New York.

Albanesi, G.L.; Bergström, S.M. 2010. Early-Middle Ordovician conodont paleobiogeography with special regard to the geographic origin of the Argentine Precordillera: A multivariate data analysis. The Geological Society of América, Special Paper 466: 119-139.

Albanesi, G.L.; Ortega, G. 2016. Conodont and graptolite bioestratigraphy of the Ordovician System of Argentina. In Stratigraphy and timescales (Montenari, M.; editor). Academic Press: 61-121.

Albanesi, G.L.; Ortega, G.; Hünicken, M.A. 1995. Conodontes y graptolitos de la Formación Yerba Loca (Arenigiano-Llandeiliano) en las quebradas de Ancaucha y El Divisadero, Precordillera de San Juan, Argentina. Boletín Academia Nacional de Ciencias 60 (3-4): 365-400. Córdoba.

Albanesi, G.L.; Hünicken, M.A.; Barnes, C.R. 1998. Bioestratigrafía de conodontes de las secuencias ordovícicas del cerro Potrerillo, Precordillera Central de San Juan, R. Argentina. Actas de la Academia Nacional de Ciencias, Tomo 12: 7-72. Córdoba.

Albanesi, G.L.; Ortega, G.; Barnes, C.R.; Hünicken, M.A. 1999. Conodont-graptolite biostratigraphy of the Gualcamayo Formation (Middle Ordovician) in the Gualcamayo-Guandacol rivers area, Argentina Precordillera. Acta Universitatis Carolinae, Geología 43 (1-2): 45-48.

Albanesi, G.L.; Carrera, M.G.; Cañas, F.L.; Saltzman, M. 2006. A proposed Global Boundary Stratotype Section and Point for the base of the Middle Ordovician Series: The Niquivil section, Precordillera of San Juan, Argentina. Episodes 29: 1-15.

Albanesi, G.L.; Ortega, G.; Monaldi, C.R. 2014. Precisiones sobre la edad de la Formación Cieneguillas (Ordovícico) en el Alto de Lipán, sierra de Los Colorados, Cordillera Oriental de Jujuy. In Congreso Geológico Argentino, No. 19: S2-1. Córdoba.

An, T.-X. 1987. The Lower Paleozoic conodonts of South China. Peking University Publication House: 1-238. Beijing.

Astini, R.A. 1992. Tectofacies ordovícicas y evolución de la Cuenca eopaleozoica de la Precordillera Argentina. Estudios Geológicos 48: 315-327.

Astini, R.A. 1994. Análisis secuencial y paleoambientes de las pelitas negras (Aloformación Gualcamayo) que suprayacen a las sucesiones carbonáticas eoordovícicas en la Precordillera Argentina. Revista de la Asociación Geológica Argentina 49: 71-84.

Astini, R.A.; Benedetto, J.L.; Vaccari, N.E. 1995. The early Paleozoic evolution of the Argentine Precordillera as a Laurentian rifted, drifted, and collided terrane: A Geodynamic model. Geological Society of America Bulletin 107: 253-273.

Astini, R.A.; Ramos, V.A.; Benedetto, J.L.; Vaccari, N.E.; Cañas, F.L. 1996. La Precordillera: un terreno exótico a Gondwana. In Congreso Geológico Argentino, No. 13, y Congreso de Exploración de Hidrocarburos, No. 3, Actas 5: 293-324. Buenos Aires.

Bagnoli, G.; Stouge, S. 1991. Paleogeographic distribution of Arenigian (Lower Ordovician) conodonts. Anais Academia Brasileira de Ciências 63 (2): 171-183.

Bagnoli, G.; Stouge, S. 1997. Lower Ordovician (Billingenian-Kunda) conodont zonation and provinces based on sections from Horns Udde, north Öland, Sweden. Bolletino della Società Paleontologica Italiana 35 (2): 109-163.

Baldis, B.A.J.; Chebli, G. 1969. Estructura profunda del área central de la Precordillera sanjuanina. In Jornadas Geológicas Argentinas, No. 4: 47-66. Mendoza.

Baldis, B.A.J.; Beresi, M. 1981. Biofacies de culminación del ciclo deposicional calcáreo del Arenigiano de oeste de Argentina. In Anais Congreso Latinoamericano de Paleontología, No. 2, 1: 11-19. Porto Alegre. 
Baldis, B.A.J.; Beresi, M.; Bordonaro, O.; Vaca, A. 1982. Síntesis evolutiva de la Precordillera Argentina. In Congreso Latinoamericano de Geología, No. 5, Actas 4: 399-445. Buenos Aires.

Baldis, B.A.J.; Bordonaro, O.; Armella, C.; Beresi, M.; Cabaleri, N.; Peralta, S.; Bastías, H. 1989. La cuenca paleozoica inferior de la Precordillera Argentina. In Cuencas sedimentarias argentinas (Chebli, G.A.; Spalletti, L.A.; editores). Serie Correlación Geológica No. 6: 101-121. Tucumán.

Barnes, C.R.; Rexroad, C.B.; Miller, J.F. 1973. Lower Paleozoic conodont provincialism. In Conodont Paleozoology (Rhodes, F.H.T.; editor). Geological Society of America, Special Paper 141: 157-190.

Benedetto, J.L. 1993. La hipótesis de la aloctonía de la Precordillera Argentina: un test estratigráfico y bioestratigráfico. In Congreso Geológico Argentino, No. 12, y Congreso de Exploración de Hidrocarburos, No. 2, Actas 3: 375-384. Mendoza.

Benedetto, J.L. 2004. The allochthony of the Precordillera ten years later (1993-2003). A new paleobiogeoraphic test of the microcontinental model. Godwana Research 7: 1027-1039.

Bergström, S.M. 1971. Conodont biostratigraphy of the Middle and Upper Ordovician of Europe and eastern North America. In Symposium on conodont biostratigraphy (Sweet, W.C.; Bergström, S.M.; editores). Geological Society of America Memory 127: 83-157.

Bergström, S.M. 1973. Ordovician conodonts. In Atlas of Palaeobiogeography (Hallam, A.; editor). Elsevier: 47-58.

Bergström, S.M.; Sweet, W.C. 1966. Conodonts from the Lexington Limestone (Middle Ordovician) of Kentucky and its lateral equivalents in Ohio and Indiana. Bulletin of American Paleontology 50 (229): 271-441.

Cañas, F.L. 1995. Estratigrafía y evolución paleoambiental de las sucesiones carbonáticas del Cámbrico tardío y Ordovícico temprano de la Precordillera Septentrional, República Argentina. Tesis Doctoral en Ciencias Geológicas (Inédito), Facultad de Ciencias Exactas, Físicas y Naturales, Universidad Nacional de Córdoba: 216 p.

Cañas, F.; Keller, M. 1993. Arrecifes y montículos arrecifales en la Formación San Juan (Precordillera Sanjuanina, Argentina): Los arrecifes más antiguos de Sudámerica. Boletín del Real Sociedad Española de Historia Natural, Sección Geológica 88 (1-4): 127-136.

Cañas, F.L.; Carrera, M.G. 2003. Precordilleran reefs. In Ordovician fossils of Argentina (Benedetto, J.L.; editor). Secretaría de Ciencia y Tecnología, Universidad Nacional de Córdoba: 131-153.
Carlorosi, J.M. 2013. La Zona de Baltoniodus triangularis (Conodonta) en el Paleozoico de la Cuenca Central Andina Sudamericana: Formación Alto del Cóndor del Norte argentino. Boletín Geológico y Minero 124: 551-62.

Carlorosi, J.; Heredia, S. 2013. The conodont Trapezognathus diprion (Lindström) in the Acoite Formation, Eastern Cordillera, Northwestern Argentina. In Conodonts from the Andes, International Conodont Symposium, No. 3 (Albanesi, G.L.; Ortega, G.; editores). Asociación Paleontológica Argentina. Publicación Especial 13: 1-4. Mendoza.

Carrera, M.G. 2003. Sponges and Bryozoans. In Ordovician Fossils of Argentina (Benendetto, J.L.; editor). Secretaría de Ciencia y Tecnología, Universidad Nacional de Córdoba: 155-185.

Carrera, M.G.; Fenoglio, F.; Albanesi, G.L.; Voldman, G.G. 2013. Conodonts, sequence stratigraphy and the rowning of the San Juan carbonate platform in the Ordovician of the Argentine Precordillera. In Conodonts from the Andes, International Conodont Symposium, No. 3 (Albanesi, G.L.; Ortega, G.; editores). Asociación Paleontológica Argentina. Publicación especial 13: 5-12. Mendoza.

Cech, N.; Carrera, M.G. 2002. Dinámica de las comunidades arenigianas de la Formación San Juan (Ordovícico), Precordillera Argentina. Ameghiniana 39: 21-40.

Christiansen, J.L.; Stouge, S. 1999. Oceanic circulation as an element in palaeogeographical reconstructions: the Arenig (Early Ordovician) as an example. Terra Nova 11 (2-3): 73-78.

Comité Argentino de Estratigrafía. 1992. Código Argentino de Estratigrafía. Asociación Geológica Argentina, B (20): 1-64.

Della Costa, G.; Albanesi, G.L. 2016. Bioestratigrafía de conodontes floianos y dapingianos (Ordovícico InferiorMedio) de la Formación San Juan en la localidad de Peña Sombría, Precordillera de La Rioja. In Congreso de la Asociación Paleontológica Argentina, Resumen: p. 38. Río Negro.

Di Prinzio, B.J.; Hünicken, M.A. 1990. Conodont fauna and biostratigraphy of the San Juan Formation (Ordovician) of Quebrada de Huaco, Jachal department, San Juan province, Argentina. In Latin American Conodont Symposium, No. 1 (Hünicken, M.A.; editor): 99-101. Córdoba.

Dubinina, S.V. 2000. Conodonts and zonal stratigraphy of the Cambrian-Ordovician boundary deposits. Transactions of the Russian Academy of Sciences, Geological Institute 517: 1-239. 
Epstein, A.G.; Epstein, J.B.; Harris, L.D. 1977. Conodont color alteration - an index to organic metamorphism. United States Geological Survey Professional Paper 995: 1-27.

Ethington, R.L.; Clark, D.L. 1981. Lower and Middle Ordovician conodonts from the Ibex area, western Millard County. Utah Brigham Young University, Geology Studies 28: 1-160.

Ethington, R.L.; Repetski, J.E. 1984. Paleobiogeographic distribution of Early Ordovician conodonts in central and western United States. In Conodont Biofacies and Provincialism (Clark, D.L.; editor). Geological Society of America, Special Paper 196: 89-101. Colorado.

Ethington, R.L.; Finney, S.C.; Miller, J.F.; Ross, R.J. Jr.; Valdes-Camin, C. 1995. Pre-meeting trip-Central Great Basin Transect. In Ordovician of the Great Basin: Fieldtrip Guidebook and Volume for the Seventh International Symposium on the Ordovician System (Cooper, J.D.; editor) 78: 1-50. California.

Feltes, N.A.; Albanesi, G.L.; Bergström, S.M. 2013. Middle Darriwilian conodont biozones from the lower member of the Las Aguaditas formation, central Precordillera of San Juan, Argentina. In Conodonts from the Andes (Albanesi, G.L.; Ortega, G.; editores), Proceedings of the International Conodont Symposium, No. 3. Asociación Paleontológica Argentina. Publicación Especial 13: 25-31. Mendoza.

Feltes, N.A.; Albanesi, G.L.; Bergström, S.M. 2016. Conodont biostratigraphy and global correlation of the middle Darriwilian-lower Sandbian (Ordovician) Las Aguaditas Formation, Precordillera of San Juan, Argentina. Andean Geology 43 (1): 60-85. doi: 10.5027/ andgeoV43n1-a04.

Finney, S.C. 2007. The parautochthonous Gondwanan origin of the Cuyania (greater Precordillera) terrane of Argentina: A re-evaluation of evidence used to support an allochthonous Laurentian origin. Geológica Acta 5 (2): 127-158.

Gutiérrez-Marco, J.C.; Albanesi, G.L.; Sarmiento, G.N.; Carlotto, V. 2008. An Early Ordovician (Floian) Conodont Fauna from the Eastern Cordillera of Perú (Central Andean Basin). Geológica Acta 6 (2): 147-160.

Heredia, S.; Mestre, A. 2011. Middle Darriwilian Conodont Biostratigraphy in the Argentine Precordillera. In Ordovician of the World (Gutiérrez Marco, J.C.; Rábano, I.; García Bellido, D.; editores). Cuadernos del Museo Geominero 14: 229-234.

Heredia, S.; Mestre, A. 2013. El conodonte darriwiliano Lenodus variabilis (Sergeeva) en la Precordillera
Central de San Juan, Argentina. Serie Correlación Geológica 29 (1): 81-92.

Hünicken, M.A. 1985. Lower Ordovician conodont biostratigraphy in Argentina. Boletín Academia Nacional de Ciencias 56 (3-4): 309-321. Córdoba.

Hünicken, M.A.; Ortega, G. 1987. Lower Llanvirn-Lower Caradoc (Ordovician) conodonts and graptolites from the Argentine Central Precordillera. In Conodonts: Investigative Techniques and Applications (Austin, R.L.; editor). Ellis Horwood Limited 7: 136-145. Chichester.

Ji, Z.; Barnes, C.R. 1994. Lower Ordovician conodonts of the St. George Group, Port au Port Peninsula, western Newfoundland, Canada. Palaeontographica Canadiana, Geological Association of Canada and Canadian Society of Petroleum Geologists 11: 1-149. Calgary.

Johnston, D.I.; Barnes, C.R. 1999. Early and Midde Ordovician (Arenig) conodontes from St. Pauls Inlet and Martin Point, Cow Head Group, Western Newfoundland, Canada. Biostratigraphy and paeoecology. Geologica et Palaeontologica 33: 21-70.

Kobayashi, T. 1937. The Cambro-Ordovician shelly faunas of South America. Faculty of Science Journal, Imperial University of Tokyo, section II, Geology, Mineralogy, Geography, Seismology 4: 369-522.

Lehnert, O. 1993. Bioestratigrafía de los conodontes arenigianos de la Formación San Juan en la localidad de Niquivil (Precordillera sanjuanina, Argentina) y su correlación intercontinental. Revista Española de Paleontología 8 (2): 153-164.

Lehnert, O. 1995. Ordovizische conodonten aus der Präkordillere westargentiniens: Ihre bedeutung für Stratigraphie und Paläogeographie. Erlanger Geologische Abhandlungen 125: 1-193.

Lehnert, O.; Miller, J.F.; Repetski, J.E. 1997. Paleogeographic significance of Clavohamulus hintzei Miller (Conodonta) and other Ibexian conodonts in an early Paleozoic carbonate platform facies of the Argentine Precordillera. Geological Society of America Bulletin 109 (4): 429-443.

Lehnert, O.; Keller, M.; Bordonaro, O. 1998. Early Ordovician conodonts from the southern Cuyania terrane (Mendoza Province, Argentina). Palaeontologica Polonica 58: 47-65.

Lehnert, O.; Stouge, S.; Brandl, P.A. 2013. Conodont biostratigraphy in the Early to Middle Ordovician strata of the Oslobreen Grup in Ny Friesland, Svalbard. Zeitschrift der Deutschen Gesellschaft für Geowissenschaften 164: 149-172.

Lemos, V.B. 1981. Conodontes ordovicianos da Formacào San Juan, Precordillera, Departamento Jáchal, Provincia 
de San Juan, República Argentina (Bioestratigrafia). In Anais Congresso Latinoamericano de Paleontologia, No. 2, 1: 31-43. Porto Alegre.

Lindström, M. 1971. Lower Ordovician conodonts of Europe. In Symposium on Conodont Biostratigraphy (Sweet, W.C.; Bergström, S.M.; editores). Geological Society of America Memoir 127: 21-61.

Löfgren, A. 1978. Arenigian and Llanvirnian conodonts from Jamtland, northern Sweden. Fossils and Strata 13: $1-129$.

Ludvigsen, R.; Westrop, S.R.; Pratt, B.R.; Tuffnell, P.A.; Young, G.A. 1986. Dual biostratigraphy: Zones and biofacies. Geoscience Canada 13 (3): 139-154.

Maciel-Mata, C.A.; Manríquez-Morán, N.; Octavio-Aguilar, P.; Sánchez-Rojas, G. 2015. El área de distribución de las especies: revisión del concepto. Acta Universitaria 25 (2): 3-19.

Mango, M.; Varini, M.; Albanesi, G. 2016. Biozonas de conodontes de la Formación San Juan (Ordovícico) y cronoestratigrafía de los cadilitos carbonáticos de la Formación Guandacol (Carbonífero) en el anticlinal de Huaco, Precordillera de San Juan. Acta Geológica Lilloana 28 (suplemento): 123-126.

Mestre, A. 2008. Revisión bioestratigráfica del techo de la Formación San Juan (Ordovícico Inferior), en la sección del Monumento a Buenaventura Luna, Precordillera Central, San Juan. Acta Geologica Lilloana 20 (2): 127-136.

Mestre, A. 2013. Middle Darriwilian conodont biostratigraphy of the Villicum range, eastern Precordillera, Argentina. Asociación Paleontológica Argentina, Publicación Especial 13: 69-72.

Mestre, A.; Beresi, M.; Heredia, S.; Nestell. G. 2013. Microfossils of the Yangtzeplacognathus crassus Zone in the middle Darriwilian of the Argentine Precordillera. In Conodonts from the Andes (Albanesi, G.L.; Ortega, G.; editors). International Conodont Symposium, No. 3, Asociación Paleontológica Argentina, Publicación Especial 13: 79-83.

Ortega, G. 1987. Las graptofaunas y los conodontes de la Formación Los Azules, cerro Viejo, Zona de Huaco, Departamento Jáchal, San Juan. Tesis Doctoral en Ciencias Geológicas (Inédito), Facultad de Ciencias Exactas, Físicas y Naturales, Universidad Nacional de Córdoba: 210 p.

Ortega, G.; Albanesi, G.L.; Ottone, E.G.; Holfeltz, G.D. 1996. Conodonts, palynomorphs and graptolites from the Los Azules Formation, Argentine Precordillera. In International conodont Symposium, ECOS, No. 6, Abstracts: p. 44. Warszawa.
Ortega, G.; Albanesi, G.L.; Frigerio, S.E. 2007. Graptoliteconodont biostratigraphy and biofacies of the Middle Ordovician cerro Viejo succession, San Juan Precordillera Argentina. Palaeogeography, Palaeoclimatology, Palaeoecology 245: 245-263.

Ortiz, A.; Zambrano, J. 1981. La Provincia geológica Precordillera Oriental. In Congreso Geológico Argentino, No. 8, 3: 59-74. San Luis.

Ottone, E.G.; Albanesi, G.; Ortega, G.; Holfeltz, G. 1999. Palynomorphs, conodonts and associated graptolites from the Ordovician Los Azules Formation, Central Precordillera, Argentina. Micropaleontology 45 (3): 225-250.

Pohler, S.M.; Barnes, C.R. 1990. Conceptual models in conodont paleoecology. Courier Forschungsinstitut Senckenberg 118: 409-440.

Pohler, S.M.L.; Orchard, M.J. 1990. Ordovician conodont biostratigraphy, western canadian cordillera. Geological Survey of Canada 90-15: 1-37.

Pyle, L.J.; Barnes, C.R. 2002. Taxonomy, evolution, and biostratigraphy of conodonts from the Kechika Formation, Skoki Formation, and Road River Group (Upper Cambrian to Lower Silurian), Northeastern British Columbia. National Reasearch, Council Research Press: 1-227. Canada.

Pratt, B.; Raviolo, M.M.; Bordonaro, O.L. 2012. Carbonate platform dominated by peloidal sands: Lower Ordovician La Silla Formation of the eastern Precordillera, San Juan, Argentina. Sedimentology: 843-866.

Ramos, V.A. 2004. Cuyania, an Exotic Block to Gondwana: Review of a Historical Success and the Present Problems. Gondwana Research 7: 1-18.

Rao, R.I.; Hünicken, M.A.; Ortega, G. 1994. Conodontes y graptolitos del Ordovícico Inferior (TremadocianoArenigiano) en el área de Purmamarca, Provincia de Jujuy, Argentina. Anais da Academia Brasileira de Ciências 66 (1): 59-83.

Rasmussen, J.A. 1998. A reinterpretation of the conondont Atlantic Realm in the late Early Ordovician (Early Llanvirn). In Proceedings of the European Conodont Symposium, ECOS, No. 6 (Szaniawski; H.; editor). Palaeontologia Polonica 58: 67-77.

Sarmiento, G.N. 1985. La Biozona de Amorphognathus variabilis-Eoplacognathus suecicus (Conodonta), Llanvirniano inferior, en el flanco oriental de la Sierra de Villicum. In Jornadas sobre Geología de Precordillera No. 1, Actas: 119-123. San Juan.

Sarmiento, G.N. 1987. Bioestratigrafía y conodontes de la Formación San Juan (Ordovícico) aflorante en el flanco oriental de la Sierra de Villicum, Provincia 
de San Juan, Argentina. Tesis Doctoral (Inédito). Universidad Nacional de Córdoba: 336 p.

Sarmiento, G.N.; Vaccari, N.E.; Peralta, S.H. 1988. Conodontes ordovícicos de La Rinconada, Precordillera de San Juan, Argentina. In Congreso Argentino de Paleontología y Bioestratigrafía, No. 4, Actas 3: 219224. Mendoza.

Serpagli, E. 1974. Lower Ordovician conodont from Precordilleran Argentina (Province of San Juan). Bolletino della Societa Paleontologica Italiana 13 (1-2): 17-98.

Serra, F.; Albanesi, G.L.; Bergström, S.M. 2013. Middle Dariwilian conodont biostratigraphy of the Las Chacritas Formation, Central Precodillera of San Juan, Argentina. In Conodonts from the Andes, International Conodont Symposium, No. 3 (Albanesi, G.L.; Ortega, G.; editores). Asociación Paleontológica Argentina. Publicación Especial 13: 109-115. Mendoza.

Serra, F.; Feltes, N.A.; Albanesi, G.L. 2017. Diversity and turnover rates of conodont species from the Middle Ordovician of the Argentine Precordillera. In International Conodont Symposium (ICOS) No. 4. Progress on conodont investigation (Liao, J.; Valenzuela-Ríos, J.I.; editores). Cuadernos del Museo Geominero 22: 277-281.

Smith, M.P. 1991: Early Ordovician conodonts of East and North. Greenland. Meddelelser om Grønland Geoscience 26: 1-81.

Soria, T.; Heredia, S.; Mestre, A.; Rodríguez, C. 2013. Conodontes floianos de la Formación San Juan en la quebrada de Talacasto, Precordillera de San Juan. Serie Correlación Geológica 29 (1): 93-106. Tucumán.

Stone, J. 1987. Review of investigative techniques used in the study of conodontes. In Conodonts: Investigative Techniques and Applications (Austin, R. L.; editor). Ellis Horwood Limited: 17-34.

Stouge, S. 2012. Middle Ordovician (late DapingianDarriwilian) conodonts from the Cow Head Group and Lower Head Formation, western Newfoundland, Canada. Canadian Journal Earth Sciences 49: 59-90.

Stouge, S.; Harper, D.A.T.; Sevastopulo, G.D.; O’Mahony, D.; Murray, J. 2016. Lower and Middle Ordovician conodonts of Laurentian affinity from blocks of limestone in the Rosroe Formation, South Mayo Trough, western Ireland and their palaeogeographic implication. Geological Journal 51 (4): 584-599.

Sweet, W.C. 1984. Graphic correlation of upper Middle and Upper Ordovician rocks, North American midcontinent province, USA. Aspects of the Ordovician System 295: 23-35.

Sweet, W.C.; Ethington, R.L.; Barnes, C.R. 1971. North American Middle and Upper Ordovician conodont faunas. In Symposium on conodont biostratigraphy (Sweet. W.C.; Bergström, S.M.; editores). Geological Society of America, Memoir 127: 163-193. Colorado.

Thomas, W.A.; Astini, R.A. 1996. The Argentine Precordillera: A traveler from the Ouachita Embayment of North Américan Laurentia. Science 273: 752-757.

Tolmacheva, T.J. 2001. Conodont biostratigraphy and diversity in the Lower-Middle Ordovician of eastern Baltoscandia (St. Petersburg region, Russia) and Kazakhstan. Summary in Ph.D. Thesis (Unpublished), Department of Earth Sciences, Historical Geology and Palaeontology Uppsala University: 40 p.

Voldman, G.G.; Albanesi, GL.; Zeballo, F.J.; Monaldi, C.R. 2013. Early Ordovician (Late Floian) conodonts from the Zenta Range, Cordillera Oriental, NW Argentina. In Conodonts from the Andes, International Conodont Symposium, No. 3 (Albanesi, G.L.; Ortega, G.; editores). Asociación Paleontológica Argentina. Publicación especial 13: 123-128.

Voldman, G.G.; Albanesi, G.L.; Ortega, G.; Giuliano, M.E.; Monaldi, C.R. 2017. New conodont taxa and biozones from the Lower Ordovician of the Cordillera Oriental, NW Argentina. Geological Journal 52 (3): 394-414.

Wang, C.-Y. 1990. Conodont biostratigraphy of China. Courier Forschungsinstitut Senckenberg 118: 591-610.

Wang, Z.-H.; Bergström, S.M. 1995. Castlemainian (Late Yushanian) to Darriwilian (Zhejiangian) conodont faunas. In The base of the Austrodentatus Zone as a level for global subdivision of the Ordovician System (Chen, X.; Bergström, S.M.; editores). Palaeoworld, Nanjing University Press 5: 86-91.

Wang, X.; Li, Z.; Chen, X.; Wang, C. 2003. The Huanghuachang section, potential as Global stratotype for the base of the Middle Ordovician Series. In Ordovician from Andes (Albanesi, G.L.; Beresi M.S.; Peralta S.H.; editores). Instituto Superior de Correlación Geológica, Serie Correlación Geológica, 17: 153-159.

Wang, X.; Stouge, S.; Erdtmann B.-D.; Chen, X.; Li, Z.; Wang; C.; Zeng, Q.; Zeng, Q.; Zhou, Z.; Chen, H. 2005. A proposed GSSP for the base of the Middle Ordovician Series: the Huanghuachang section, Yichang, China. Episodes 28 (2): 105-117.

Zhen, Y.Y.; Percival, I.G. 2003. Ordovician conodont biogeography-reconsidered. Lethaia 36: 357-370. 